\title{
Probing Scrambling Using Statistical Correlations between Randomized Measurements
}

\author{
B. Vermersch, ${ }^{1,2, *}$ A. Elben, ${ }^{1,2}$ L. M. Sieberer, ${ }^{1,2}$ N. Y. Yao, ${ }^{3,4}$ and P. Zoller ${ }^{1,2}$ \\ ${ }^{1}$ Center for Quantum Physics, University of Innsbruck, Innsbruck A-6020, Austria \\ ${ }^{2}$ Institute for Quantum Optics and Quantum Information, Austrian Academy of Sciences, \\ Innsbruck A-6020, Austria \\ ${ }^{3}$ Department of Physics, University of California, Berkeley, California 94720, USA \\ ${ }^{4}$ Materials Science Division, Lawrence Berkeley National Laboratory, Berkeley, California 94720, USA
}

(Received 12 December 2018; published 27 June 2019)

\begin{abstract}
We propose and analyze a protocol to study quantum information scrambling using statistical correlations between measurements, which are performed after evolving a quantum system from randomized initial states. We prove that the resulting correlations precisely capture the so-called out-of-time-ordered correlators and can be used to probe chaos in strongly interacting, many-body systems. Our protocol requires neither reversing time evolution nor auxiliary degrees of freedom, and it can be realized in state-of-the-art quantum simulation experiments.
\end{abstract}

DOI: 10.1103/PhysRevX.9.021061

Subject Areas: Atomic and Molecular Physics, Quantum Physics, Quantum Information

\section{INTRODUCTION}

Recent developments in quantum simulation have enabled the remarkable ability to interrogate and control atomic, molecular, and ionic degrees of freedom (d.o.f.) in lattice experiments with single-site resolution [1-4]. In atomic Hubbard models with bosonic and fermionic atoms in optical lattices, a quantum gas microscope provides us with single-shot spatial- and spin-resolved images of atomic densities. By averaging over many images, this allows one to extract spatial and spin equal-time correlation functions, which reveal unique properties of (non)equilibrium quantum phases [5-8]. In spin models, as realized with trapped ions [9,10], Rydberg atoms [11-14], and superconducting qubits [15-18], the state of the spins (qubits) can be measured in a given standard basis in single-shot measurements with high fidelity and with high repetition rates. Building on these tools, we develop below quantum protocols to measure many-body observables from analyzing statistical cross-correlations between such quantum images representing different runs of an experiment. We see that this provides us with simple, generic, and robust techniques to extract many-body observables, which are challenging to access otherwise within existing experimental setups. In particular, we develop novel protocols for out-of-time-ordered correlators (OTOCs), which are

\footnotetext{
*benoit.vermersch@1pmmc.cnrs.fr
}

Published by the American Physical Society under the terms of the Creative Commons Attribution 4.0 International license. Further distribution of this work must maintain attribution to the author(s) and the published article's title, journal citation, and DOI. time-dependent quantities that cannot be measured directly as a standard time-ordered correlation function. OTOCs represent a key quantity to diagnose quantum chaos and enable one to understand how quantum information propagates, and "scrambles" [19], in close connection to the notion of entanglement spreading [20-23].

OTOCs have been introduced to characterize quantum dynamics, described by a unitary time-evolution operator $U(t)$ in terms of the complexity of Heisenberg operators $W(t)=U^{\dagger}(t) W U(t)$. For chaotic dynamics, even an initially "simple" and local Hermitian operator $W$ rapidly becomes complex and nonlocal. As a consequence, after a short time, $W(t)$ is delocalized and no longer commutes with an initially nonoverlapping local operator $V$. The degree of noncommutativity, or equivalently the scrambling of $W(t)$, is quantified by the OTOC, which takes the form

$$
O(t)=\operatorname{Tr}\left(\rho W(t) V^{\dagger} W(t) V\right) / \operatorname{Tr}\left(\rho W(t)^{2} V^{\dagger} V\right)
$$

with $\rho$ the initial quantum state. Note that this definition ensures that $O(t)=1$ when $W(t)$ and $V$ commute. In the following, we focus on the "infinite-temperature" OTOC [22-26] for which $\rho=I / \mathcal{N}_{\mathcal{H}}$, with $I$ the identity operator and $\mathcal{N}_{\mathcal{H}}$ the Hilbert space dimension. In Sec. V, we discuss extensions of our approach to thermal states. The time dependence of $O(t)$ can distinguish between different classes of scrambling, ranging from "fast scrambling" in models with holographic duals [27-32] and chaotic many-body spin systems $[19,22,23,33,34]$, to "slow scrambling" characteristic of many-body localization (MBL) [24-26,35]. These theoretical insights raise the question of how to experimentally measure $O(t)$, despite the 
peculiar time order inherent in its definition. A first option to measure $O(t)$ consists in implementing time-reversal operations [21,36-39] or using auxiliary quantum systems [40]. The first measurements of OTOCs were realized using this approach in systems with few d.o.f. [41], but also in a trapped ion setup with infinite-range interactions [42]. However, protocols based on realizing time-reversal operations remain an experimental challenge for many experimental platforms-like Hubbard systems or systems with local interactions. For such protocols, recent studies have also shown that decoherence can "mimick" the effect of scrambling, and they have developed [39,43] and realized [44] implementations involving auxiliary d.o.f. to distinguish the two effects.

In contrast, a unique feature of our protocols to measure OTOCs via statistical correlations is that they do not rely on time-reversal operations nor the presence of an ancillary system. In addition, we show that OTOCs extracted from statistical averages provide the key advantage to be naturally robust against various forms of decoherence and experimental noise, including depolarization and readout errors. As a consequence, our protocols can be realized in any state-of-the-art AMO $[2,3,45]$ or superconducting qubit platforms [4], and they can be used as experimental probes of scrambling in many-body systems.

Our work presents two key results related to two protocols. First, we introduce the global protocol and demonstrate the exact equivalence between the OTOC $O(t)$, as defined above, and the statistical correlations obtained from initial states, which are randomized with a global unitary operator $u$ for the total many-body system. We then present the local protocol, which consists of an experimentally simpler approach for spin systems, where the initial state is randomized with local unitary operations and where the statistical correlations also give access to $O(t)$.

This paper is organized as follows. Section II presents the main results of this paper by describing the two protocols to measure $O(t)$ with local and random unitaries. Section III gives different physical examples, accessible to current AMO and solid-state platforms. Finally, we discuss in Sec. IV the role of statistical errors and imperfections, and we identify, in particular, the different situations (depolarization, readout errors) where the protocol is not affected.

\section{PROTOCOLS MAPPING STATISTICAL CORRELATIONS TO OTOCS}

In this section, we present and illustrate both the global and local protocols to measure OTOCs via random measurements. We consider a system $\mathcal{S}$ associated with a Hilbert space of dimension $\mathcal{N}_{\mathcal{H}}$. This system can be, for example, a set of atoms described by a Hubbard model or an ensemble of spin-1/2 as shown in Fig. 1(a). In the following, we also assume the operator $V$ to be unitary and the operator $W$ to be Hermitian and traceless $[\operatorname{Tr}(W)=0]$. Note that these conditions do not restrict the ability of (a)
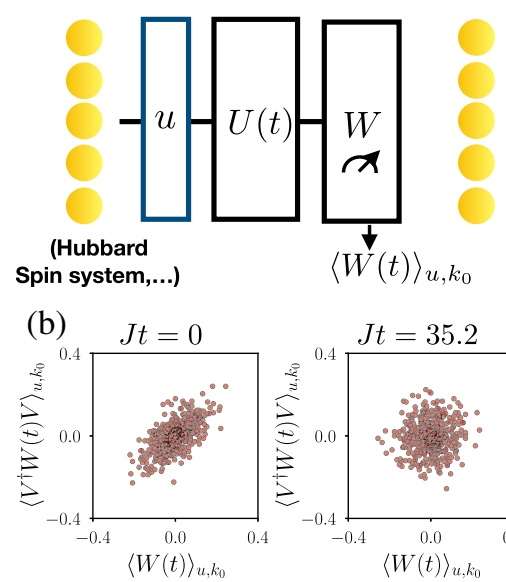

Global protocol

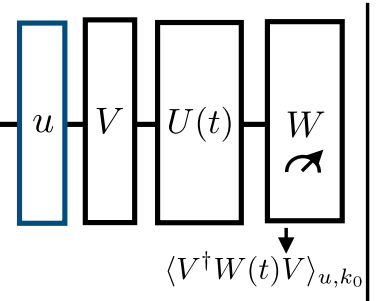

(c)

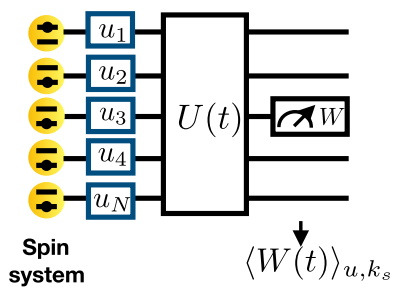

Local protocol
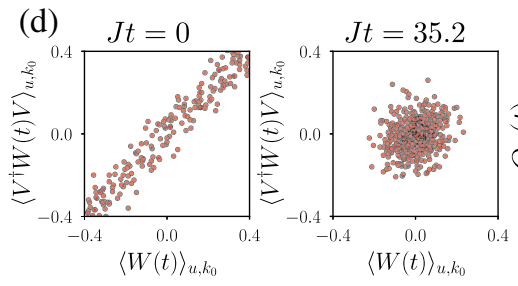

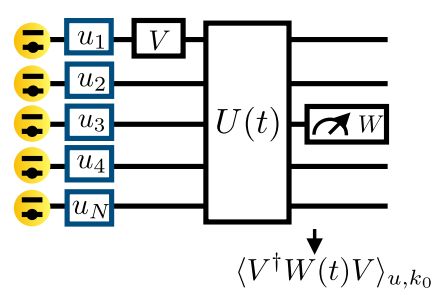

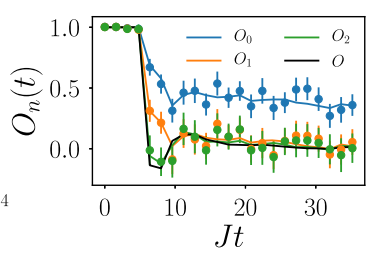

FIG. 1. Probing scrambling via statistical correlations in a spin system. (a) The global protocol consists in separately measuring $\langle W(t)\rangle_{u, k_{0}}$ and $\left\langle V^{\dagger} W(t) V\right\rangle_{u, k_{0}}$ to obtain the OTOC $O(t)$. (b) Numerical simulations of the protocol for the kicked Ising model with parameters $h_{x}=J, h_{z}=0.809 J, J T=1.6, N=8$, and $j=3$. The first two panels show the statistics of the measurement at $J t=0$ and $J t=35.2$. In the third panel, the exact OTOC $O(t)$ is shown as a solid line, and the points represent the statistical correlations obtained from $N_{u}=500$ unitaries and $N_{M}=500$ projective measurements (per unitary), with error bars placed at $\pm 2 \sigma$ calculated from the jackknife resampling method. (c) Local protocol using local random unitaries. (d) Same as (b) with the local protocol, extracting the modified OTOC $O_{n}(t)$ (calculated exactly, colored lines) from the statistical correlations (circles with error bars). Convergence to the OTOC $O(t)$ (black line) is achieved for low index $n \sim 2$. Throughout this work, we use unbiased estimators for the normalization terms $\mathcal{D}^{(\mathrm{G}, \mathrm{L})}$. 
OTOCs to describe scrambling: For spin systems, we consider, as examples, $W$ and $V$ to be local Pauli operators, which are particularly relevant in this context [22,23]. We also give examples below that are relevant to probe scrambling in Hubbard systems.

Our two protocols are illustrated in Fig. 1. The first protocol described in Sec. II A relies on global random unitaries $u$ from the circular unitary ensemble $\operatorname{CUE}\left(\mathcal{N}_{\mathcal{H}}\right)$ [46] or from a unitary 2-design [47]. As illustrated below for a Bose-Hubbard (BH) system, such random unitaries can be realized in generic interacting models using timedependent disorder [48-50]. The second protocol presented in Sec. II B considers spin systems with individual spin control, which allows us to drastically simplify the experimental task by replacing the global random unitaries by local random unitaries $u=u_{1} \otimes u_{2} \otimes \ldots \otimes u_{N}, u_{i} \in$ CUE(2), acting on single spins $i=1, \ldots, N$. Note that such local random unitaries have recently been realized with high fidelity with trapped ions [10,51].

We find it convenient to present both protocols as experimental recipes to measure the statistical correlations. In each case, we then mathematically relate the correlations to $O(t)$.

\section{A. Global protocol}

Experimental protocol.-The protocol consists of the following steps, as illustrated in Fig. 1(a).

(i) We prepare an arbitrary state $\left|k_{0}\right\rangle$ and apply a global random unitary $u$ to obtain $\left|\psi_{u, k_{0}}\right\rangle=u\left|k_{0}\right\rangle$. The randomized state $\left|\psi_{u, k_{0}}\right\rangle$ is the starting point for two independent experiments:

(ii.a) In the first experiment, we evolve the system in time with $U(t)$ and perform a measurement of the expectation value of $W$. The time-evolution operator $U(t)$ can be generated, for instance, from a static Hamiltonian $U(t)=e^{-i H t}$, from Floquet evolution, or from a quantum circuit operating on qubits, depending on the type of system under study. We repeat steps (i) and (ii.a) with the same random unitary $u$ to measure $\langle W(t)\rangle_{u, k_{0}}=$ $\left\langle\psi_{u, k_{0}}|W(t)| \psi_{u, k_{0}}\right\rangle$, as illustrated in Fig. 1(a).

(ii.b) In the second experiment, we prepare again $\left|\psi_{u, k_{0}}\right\rangle$ and apply the unitary $V$. This operation is followed by the time evolution with $U(t)$ and a measurement of $W$. We repeat this sequence to obtain $\left\langle V^{\dagger} W(t) V\right\rangle_{u, k_{0}}=\left\langle\psi_{u, k_{0}}\left|V^{\dagger} W(t) V\right| \psi_{u, k_{0}}\right\rangle$, as shown in Fig. 1(a).

(iii) Finally, we repeat steps (i) and (ii) for different random unitaries. The OTOC $O(t)$, as defined in Eq. (1), is then obtained from the statistical correlations

$$
O(t)=\frac{1}{\mathcal{D}^{(\mathrm{G})}} \overline{\langle W(t)\rangle_{u, k_{0}}\left\langle V^{\dagger} W(t) V\right\rangle_{u, k_{0}}}
$$

between the measurement outcomes $\langle W(t)\rangle_{u, k_{0}}$ and $\left\langle V^{\dagger} W(t) V\right\rangle_{u, k_{0}}$ of (ii.a) and (ii.b), respectively. Here, $\cdots$ denotes the ensemble average over random unitaries $u$, and $\mathcal{D}^{(\mathrm{G})}=\overline{\langle W(t)\rangle_{u, k_{0}}^{2}}$ is a normalization term.

Proof and illustration.-Equation (2) can be proven using the 2-design identities, which provide analytical expressions for the statistical correlations between the matrix elements of $u$ [52],

$$
\begin{aligned}
& \overline{u_{m_{1}, n_{1}} u_{m_{1}^{\prime}, n_{1}^{\prime}}^{*} u_{m_{2}, n_{2}} u_{m_{2}^{\prime}, n_{2}^{\prime}}^{*}} \\
& =\frac{\delta_{m_{1}, m_{1}^{\prime}} \delta_{m_{2}, m_{2}^{\prime}} \delta_{n_{1}, n_{1}^{\prime}} \delta_{n_{2}, n_{2}^{\prime}}+\delta_{m_{1}, m_{2}^{\prime}} \delta_{m_{2}, m_{1}^{\prime}} \delta_{n_{1}, n_{2}^{\prime}} \delta_{n_{2}, n_{1}^{\prime}}}{\mathcal{N}_{\mathcal{H}}^{2}-1} \\
& \quad-\frac{\delta_{m_{1}, m_{1}^{\prime}} \delta_{m_{2}, m_{2}^{\prime}} \delta_{n_{1}, n_{2}^{\prime}} \delta_{n_{2}, n_{1}^{\prime}}+\delta_{m_{1}, m_{2}^{\prime}} \delta_{m_{2}, m_{1}^{\prime}} \delta_{n_{1}, n_{1}} \delta_{n_{2}, n_{2}{ }^{\prime}}}{\mathcal{N}_{\mathcal{H}}\left(\mathcal{N}_{\mathcal{H}}^{2}-1\right)},
\end{aligned}
$$

with $\delta$ the Kronecker delta. In order to simplify the proofs, in this work we use a diagrammatic representation of Eq. (3), where the contractions of the different indices are represented by lines [53], as shown in Fig. 2(a). This representation allows us to prove in Fig. 2(b) the identity

$$
\begin{aligned}
& \overline{\langle W(t)\rangle_{u}\left\langle V^{\dagger} W(t) V\right\rangle_{u}}=c \sum_{\tau=I, \text { swap }} \operatorname{Tr}\left[\tau\left(W(t) \otimes V^{\dagger} W(t) V\right)\right] \\
& =c \operatorname{Tr}\left[W(t) V^{\dagger} W(t) V\right] \text {, } \\
& \text { (a) } \\
& \overline{\bar{m}_{1}} u_{\overline{n_{1}}} \overline{n_{1}^{\prime}} u_{\overline{m_{1}^{\prime}}}^{\dagger} \bar{m}_{2}^{u} \overline{n_{2}} \overline{n_{2}^{\prime}} u_{\overline{m_{2}^{\prime}}}^{\dagger} \\
& =\frac{1}{\mathcal{N}_{\mathcal{H}}^{2}-1}[\circlearrowleft \curvearrowright+\Omega \\
& +\frac{-1}{\mathcal{N}_{\mathcal{H}}\left(\mathcal{N}_{\mathcal{H}}^{2}-1\right)}[\circlearrowleft \frown \circlearrowleft+\curvearrowright \\
& \text { (b) } \\
& \overline{\langle A\rangle_{u}\langle B\rangle_{u}}=\overline{\square u-\rho_{0}-u^{\dagger}-A} \overline{\square u-\rho_{0}-u^{\dagger}-B} \\
& =\frac{1}{\mathcal{N}_{\mathcal{H}}^{2}-1}[\overbrace{\rho_{0}} \overbrace{\rho_{0}}{ }_{-B}
\end{aligned}
$$

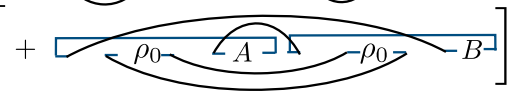

$$
\begin{aligned}
& +\frac{-1}{\mathcal{N}_{\mathcal{H}}\left(\mathcal{N}_{\mathcal{H}}^{2}-1\right)}[0 \\
& +\overbrace{\left.\left.\rho_{0}-A\right\lrcorner \square \rho_{0}>B\right]} \\
& =c \sum_{\tau \in I, \text { Swap }} \underset{\tau(A \otimes B)}{\tau(A \in I, \text { Swap }}=c \sum_{\tau i}[\tau(A \otimes B)]=c \operatorname{Tr}(A B)
\end{aligned}
$$

FIG. 2. Identities for our protocol with global unitaries. (a) Diagrammatic representation of the 2-design identities of the CUE Eq. (3). (b) Correlations between two measurements with $A=W(t), B=V^{\dagger} W(t) V$. Here, we have introduced the notation for the initial pure density matrix $\rho_{0}=\left|k_{0}\right\rangle\left\langle k_{0}\right|$. 
with $c=\left[\mathcal{N}_{\mathcal{H}}\left(\mathcal{N}_{\mathcal{H}}+1\right)\right]^{-1}$. The trace in the first line is performed over an extended Hilbert space $\mathcal{H} \otimes \mathcal{H}$, where $\mathcal{H}$ is the Hilbert space of dimension $\mathcal{N}_{\mathcal{H}}$, and the swap operator is $\operatorname{swap}\left(|k\rangle \otimes\left|k^{\prime}\right\rangle\right)=\left|k^{\prime}\right\rangle \otimes|k\rangle$ for each pair of states $|k\rangle,\left|k^{\prime}\right\rangle$. The condition of traceless operator $\operatorname{Tr}[W(t)]=0 \quad$ and the identity $\operatorname{Tr}[\operatorname{swap}(W(t) \otimes$ $\left.\left.V^{\dagger} W(t) V\right)\right]=\operatorname{Tr}\left[W(t) V^{\dagger} W(t) V\right]$ yield the second line of Eq. (4). Finally, to conclude our proof, we use the same identity with $V \rightarrow I$ to prove that the denominator in Eq. (2) reduces to $c \operatorname{Tr}\left[W^{2}(t)\right]$.

As an illustration, we present in Fig. 1(b) the intuitive physical picture behind this result [based on realizing $U(t)$ for the kicked Ising model; see caption and text below]. At $t=0$, the measurement of $W(0)=W=\sigma_{j}^{z}(j>1)$ is not affected by the operator $V=\sigma_{1}^{z}$, which distinguishes the two initial states $\left|\psi_{u, k_{0}}\right\rangle$ and $V\left|\psi_{u, k_{0}}\right\rangle$. Indeed, $[V, W(0)]=0$ and hence $\langle W(0)\rangle_{u, k_{0}}=\left\langle V^{\dagger} W(0) V\right\rangle_{u, k_{0}}$, implying perfect correlations, i.e., $O(0)=1$ [up to shot noise errors; see below and Fig. 1(b), left panel]. At later times (middle panel), when $[V, W(t)] \neq 0$ due to the spreading of $W(t)$, the value of $\left\langle V^{\dagger} W(t) V\right\rangle_{u, k_{0}}$ becomes decorrelated from $\langle W(t)\rangle_{u, k_{0}}$. Note that in analogy to our approach, the distance between a quantum state and a (physical) copy, which is perturbed at $t=0$ by the operator $V$, has been proposed to numerically detect scrambling $[54,55]$.

Measurement budget and statistical errors.-In an experiment, a finite number of $N_{u}$ random unitaries is realized to measure $O(t)$ based on Eq. (2). Furthermore, the operator $W$ is measured via a finite number of projective measurements $N_{M}$ per unitary. The total number of measurements leading to an estimation of the OTOC is therefore $N_{\text {meas }}=N_{u} N_{M}$. The finite value of $N_{\text {meas }}$ will lead to statistical errors. To illustrate this aspect, we compare in Fig. 1(b) the time evolution of $O(t)$ with the estimation obtained from a finite realistic number of measurements [10] (circles with statistical error bars). We analyze the role of statistical errors in more detail in Sec. IV.

Implementation of random unitaries. - One of the experimental challenges in the protocol presented above consists in generating, with high fidelity, global random unitaries $u$ satisfying the 2-design properties. In quantum simulators implementing Hubbard or spin models, this can be done using random quenches based on time-dependent disorder potentials (see Refs. $[49,50]$ and the example in Sec. III A). We now proceed to describe an experimentally significantly simpler protocol for spin systems, which only requires us to generate local random unitaries acting on individual spins. These unitaries can be realized by combining local rotations along a fixed axis of the Bloch sphere, say, the $z$ axis, with global rotations along an orthogonal direction, for instance, the $x$ axis $[10,51]$; they can therefore be implemented in present qubit experiments with single-site control, e.g., with trapped ions [2], Rydberg atoms [3], or superconducting qubits [4].

\section{B. Local protocol}

The protocol.-We now describe our protocol based on local unitaries. The main difference compared to the protocol presented in Sec. II A is that we need to consider an ensemble $E_{n}=\left\{\left|k_{0}\right\rangle, \ldots\right\}$ of initial states, instead of a single one $\left|k_{0}\right\rangle$, in order to obtain a mapping between statistical correlations and OTOCs. The states $\left|k_{s}\right\rangle=$ $\left|k_{s}^{(1)}, k_{s}^{(2)}, \ldots\right\rangle$ that we consider are written as product states in a standard fixed basis, i.e., $k_{s}^{(i)}=\uparrow, \downarrow$, and can be easily prepared in an experiment with single-site control.

The protocol consists of the following steps:

(i) We prepare the first initial state $\left|k_{0}\right\rangle,(s=0)$, and apply the local unitary $u=u_{1} \otimes \ldots u_{N}$, cf. Fig. 1(c), to prepare $\left|\psi_{u, k_{0}}\right\rangle$. Here, each local unitary $u_{i}$ is drawn independently from a unitary 2-design, such as the circular unitary ensemble CUE(2).

(ii.a) In the first experiment, we measure $W$ after evolving the system in time with $U(t)$. Repeating steps (i) and (ii.a) with the same random unitary $u$ gives us access to $\langle W(t)\rangle_{u, k_{0}}=\left\langle\psi_{u, k_{0}}|W(t)| \psi_{u, k_{0}}\right\rangle$.

(ii.b) In the second experiment, we proceed as in (ii.a), but we apply $V$ before the time evolution with $U(t)$. This method gives us access to $\left\langle V^{\dagger} W(t) V\right\rangle_{u, k_{0}}$.

(iii) We repeat steps (i) and (ii.a) for the other initial states $\left|k_{s}\right\rangle \in E_{n}$, using the same random unitary $u$, in order to obtain $\langle W(t)\rangle_{u, k_{s}}$, for each $k_{s} \in E_{n}$.

(iv) Finally, we repeat steps (i)-(iii) for different random unitaries. From these measurements, we can construct the statistical correlations

$$
O_{n}(t)=\frac{1}{\mathcal{D}_{n}^{(\mathrm{L})}} \sum_{k_{s} \in E_{n}} c_{k_{s}} \overline{\langle W(t)\rangle_{u, k_{s}}\left\langle V^{\dagger} W(t) V\right\rangle_{u, k_{0}}},
$$

with $\mathcal{D}_{n}^{(\mathrm{L})}=\sum_{k_{s} \in E_{n}} c_{k_{s}} \overline{\langle W(t)\rangle_{u, k_{s}}\langle W(t)\rangle_{u, k_{0}}}$, and weights $c_{k_{s}}$. Measurement budget and convergence aspects.For this protocol, the number of measurements is $N_{\text {meas }}=N_{u} N_{M}\left(\left|E_{n}\right|+1\right)$, with $\left|E_{n}\right|$ the cardinal of $E_{n}$, i.e., the number of initial states $k_{s}$. In the following, we show how to choose the ensembles $E_{n}$ and weights $c_{k_{s}}$ so that the correlations $O_{n}(t)$ represent a converging series, indexed by $n$, of "modified OTOCs" approximating $O(t)$. The low-order OTOCs $O_{0,1, \ldots}(t)$, which correspond to small numbers of initial states $\left(\left|E_{n}\right|=1,2, \ldots\right)$ to sample and are thus the easiest quantities to access experimentally, provide generically good approximations of $O(t)$. We also prove, in the other limit $n=N$, the exact relation $O_{N}(t)=O(t)$.

Introducing the modified OTOCs.-Here, for concreteness, we consider $V$ to be a Pauli operator on the first site $i=1$. For each value of $n=0, \ldots, N$, we then define the 
ensemble $E_{n}$ as the set of all $2^{n}$ configurations $\left|k_{s}\right\rangle$, such that only the states of the first $n$ spins can differ from the ones of the reference state $\left|k_{0}\right\rangle$, i.e., $k_{s}^{(i>n)}=k_{0}^{(i)}$ for $i>n$. For instance, for $n=0$ ( $n=1$, respectively), which we study in detail below, the ensemble $E_{0}=\left\{\left|k_{0}\right\rangle\right\}$ $\left(E_{1}=\left\{\left|k_{0}\right\rangle, \sigma_{1}^{x}\left|k_{0}\right\rangle\right\}\right)$ is represented by a single state (just two states). As proven in the Appendix A, by choosing the weights $c_{k_{s}}=(-1 / 2)^{d\left[k_{0}, k_{s}\right]}$, with $d\left[k_{0}, k_{s}\right]$ the Hamming distance (the number of spin flips between $\left|k_{0}\right\rangle$ and $\left|k_{s}\right\rangle$ ), we can relate the statistical correlations $O_{n}(t)$ to modified OTOCs,

$$
O_{n}(t)=\frac{\sum_{A, B_{n} \subseteq A} \operatorname{Tr}_{A}\left(W(t)_{A}(V W(t) V)_{A}\right)}{\sum_{A, B_{n} \subseteq A} \operatorname{Tr}_{A}\left(W(t)_{A} W(t)_{A}\right)},
$$

which converge to $O(t)$ for $n=N$ [56]. Here, the sums in the numerator are performed over all partitions $A$, which include the set $B_{n}=\{1, \ldots, n\}$ of the first $n$ spins (for $n=0, B_{0}=\varnothing$ is empty), and the traces are performed over reduced operators $W_{A}(t)=\operatorname{Tr}_{\mathcal{S}-A}[W(t)]$ and $(V W(t) V)_{A}=\operatorname{Tr}_{\mathcal{S}-A}(V W(t) V)$. The modified OTOCs $O_{n}(t)$ are thus sums of out-of-time-ordered functions of the different reduced operators $W(t)_{A},(V W(t) V)_{A}$.

Properties of the modified OTOCs and illustrations. The identity Eq. (6) shows that the index $n$ plays the role of a spatial resolution controlling how $O_{n}(t)$ approximates $O(t)$. For all contributing partitions $A,\{1 \ldots n\} \subseteq A$, the information about the first $n$ spins is preserved when reducing the operators $W(t) \rightarrow W(t)_{A} \quad V W(t) V \rightarrow$ $(V W(t) V)_{A}$. In particular, for the maximal spatial resolution $n=N$, statistical correlations are exactly the OTOC $O_{N}(t)=O(t)$. In the opposite case of $n=0$, all partitions $A \subseteq \mathcal{S}$ of the system contribute to $O_{0}(t)$. For $O_{1}(t)$, the information related to the support of $V$ (here, the first site) is "resolved" so that we can expect a better approximation to $O(t)$, and so on for $n=2,3, \ldots$. Note that our construction of the sets $E_{n}$ can be generalized easily to other positions of $V$ and also to multisite operators.

An illustration of this protocol is shown in Fig. 1(d) [compare to Fig. 1(b)], where we show $O_{n}(t)$ (solid lines) and the corresponding statistical correlations obtained by simulating the protocol numerically. For $n=0$, the modified OTOC captures the scrambling time as $O(t)$ but saturates to a nonzero value at long times. For $n=1,2,3, \ldots$, the values of $O_{n}(t)$ are in good quantitative agreement with $O(t)$. We also note that for short times, the local protocol has an advantage compared to the global protocol in terms of statistical errors, as we explain below.

\section{REALIZATIONS OF THE PROTOCOLS IN DIFFERENT PHYSICAL SCENARIOS}

This section is devoted to physical examples that can be accessed with our protocol. In Sec. III A, we show how to apply the global protocol in an atomic Bose-Hubbard system. We then focus on the local protocol and analyze, for chaotic (Sec. III B), many-body localized (Sec. III C), and long-range spin models (Sec. III D), the behavior of modified OTOCs $O_{n}(t)$. We analyze, in particular, both via analytical models and numerical simulations, the convergence properties of $O_{n}(t)$ to $O(t)$.

\section{A. Implementation of the global protocol in a Bose-Hubbard chain}

We now present an example to illustrate the different steps of the protocol with global unitaries and consider the situation of scrambling dynamics of the BH chain [21], with $U(t)=\exp \left(-i H_{\mathrm{BH}} t\right)$,

$H_{\mathrm{BH}}=-J \sum_{i=1}^{N-1}\left(a_{i+1}^{\dagger} a_{i}+\right.$ H.c. $)+\frac{U_{\mathrm{int}}}{2} \sum_{i=1}^{N} n_{i}\left(n_{i}-1\right)$,

with $a_{i}$ bosonic operators and $n_{i}=a_{i}^{\dagger} a_{i}$. We consider here the OTOC dynamics for the unitary $V=\exp \left(-i \pi a_{1}^{\dagger} a_{1}\right)$ and the traceless observable $W=n_{j+1}-n_{j}$.

We first illustrate the mapping Eq. (2) in Fig. 3 by comparing $O(t)$ [panel (a)] and the corresponding statistical correlations [panel (b)], where $N_{u}=1000$ random unitaries were sampled numerically from the CUE. Assuming here no projection noise $N_{M} \rightarrow \infty$, we obtain a very good agreement between the two quantities. Note, as in the case of the spin models described below, the
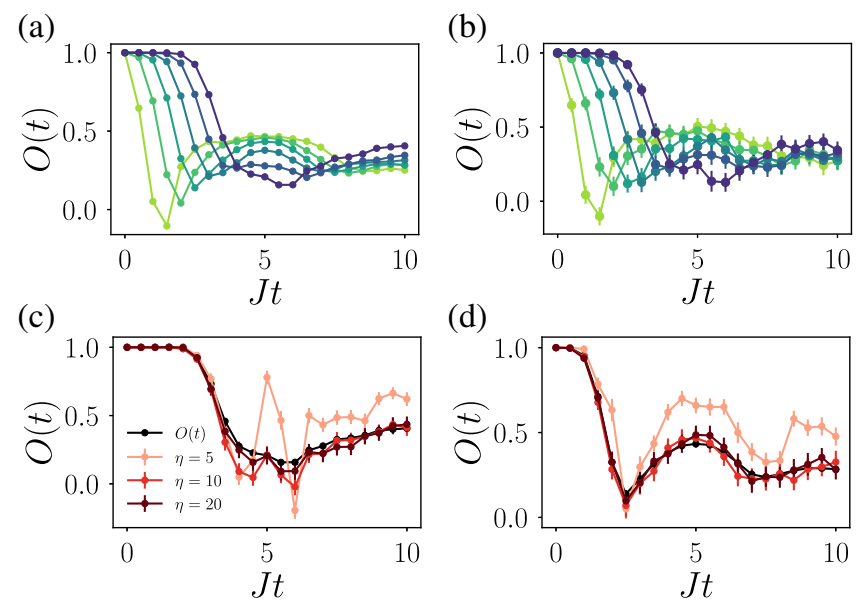

FIG. 3. Scrambling in the BH chain. (a) OTOC $O(t)$ for different positions $j=2, \ldots, N-1$ (green to purple) of the operator $W$ (see text). (b) $O(t)$ estimated with $N_{u}=1000$ unitaries $u$ sampled numerically from the Haar measure. (c,d) Comparison between $O(t)$ and corresponding estimations via statistical correlations, where $u$ is prepared via $\eta$ random quenches, with $j=7$ (c) and $j=4(\mathrm{~d})$, and $N_{u}=1000$. Here, $\Delta_{j}^{(m)}$ is sampled from a uniform distribution of width $2 J$, and the quench time is $T=1 / J$. For all panels, we consider $N=8$ lattice sites with $\left|k_{0}\right\rangle=$ $|10101010\rangle$ in the number basis, and $U_{\text {int }}=2 J$. In panels (b)-(d), error bars at 2 standard deviations are calculated using the jackknife resampling method. Here, we consider $N_{M} \rightarrow \infty$. 
characteristic "scrambling time" for the OTOCs varies essentially linearly with the position of the operators, showing the existence of a "butterfly" velocity.

We now discuss the physical realization of the global random unitaries $u$. Unitaries satisfying the required 2-design properties can be generated using the same Hamiltonian $H_{\mathrm{BH}}$ subject to a sequence of $\eta$ random quenches [49,50],

$$
u=\prod_{m=1}^{\eta} \exp \left(-i T\left[H_{\mathrm{BH}}+\sum_{j} \Delta_{j}^{(m)} n_{j}\right]\right),
$$

with $\Delta_{j}^{(m)}$ a random disorder potential, which is reinitialized for each quench $m$, and the quench time $T$. As shown in Refs. [49,50], such random quenches efficiently generate, in each particle number sector, unitaries satisfying the required 2-design properties of the CUE, after a time $\eta T \approx N$. This case is illustrated in Figs. 3(c) and 3(d) for two different operator $W$ positions: For $\eta \geq N$, the generated $u$ converges to the CUE (with respect to the required 2-design properties), and therefore, the measured statistical correlations coincide within the statistical error bars with $O(t)$.

\section{B. Chaotic dynamics with modified OTOCs}

In the rest of this section, we focus on spin models with OTOCs measured by local unitaries. To illustrate the ability of the modified OTOCs $O_{n}(t)$, Eq. (6), to approximate $O(t)$, we first consider scrambling in chaotic spin models, which is characterized by two key features: The support of an operator $W(t)$ that is initially localized grows ballistically with a "butterfly velocity" $v_{B}$, and the operator front traveling at $v_{B}$ broadens diffusively $[19,22,23]$. The ballistic growth can be captured by a simple phenomenological model, which assumes that $U(t)$ takes the form $U(t)=U[L(t)]_{1} \otimes \ldots \otimes U[L(t)]_{N / L(t)}$, where the Haar random unitaries $U[L(t)] \in \operatorname{CUE}\left(2^{L(t)}\right)$ describe scrambling on a linearly growing scale $L(t)=1+$ floor $\left(v_{B} t\right)$. For $W=\sigma_{j}^{z}$ and $V=\sigma_{1}^{z}$, we obtain, in leading order in $L(t) \gg 1[33]$,

$O(t)=1 \quad[j>L(t)] \quad O(t)=-\frac{1}{4^{L(t)}} \quad[j \leq L(t)]$,

and, as shown in Appendix B,

$$
\begin{aligned}
& O_{0}(t)=1[j>L(t)] \quad O_{0}(t)=\frac{1}{3}[j \leq L(t)], \\
& O_{1}(t)=1[j>L(t)] \quad O_{1}(t)=-\frac{1}{2^{L(t)+1}}[j \leq L(t)],
\end{aligned}
$$

Here, $O(t)$ represents the average OTOC over the unitaries $U$, whereas the expression for $O_{n}(t)$ corresponds to including the sampling over $U$ in the ensemble average ‥ [57]. All OTOCs $O(t)=O_{0,1}(t)=1$ thus coincide at short times when $W(t)$ and $V$ commute exactly. At the scrambling time
$t_{B}=r / v_{B}(r=j-1), O(t)$ and $O_{0,1}(t)$ exhibit a sharp drop. For $t>t_{B}, O(t)$ and $O_{1}(t)$ are exponentially suppressed, while $O_{0}(t)$ converges to $1 / 3$.

We now confront these analytical predictions with numerical simulations of the kicked Ising model, which is an example of a chaotic spin model [22],

$$
U(m T)=\left[e^{-i(T / 2)\left(\sum_{i} J \sigma_{i}^{z} \sigma_{i+1}^{z}+\sum_{i} h_{z} \sigma_{i}^{z}\right)} e^{-i(T / 2) h_{x} \sum_{i} \sigma_{i}^{\chi}}\right]^{m},
$$

with $m$ a positive integer and $T$ the period of the Floquet system. Throughout this work, we use open boundary conditions $(\mathrm{OBC})$. The results are shown in Fig. 4, where we compare the modified OTOCs for $n=0,1,2,3$, 4 with $O(t)$. In panel (a), corresponding to an operator position $j=4$, the scrambling dynamics described by $O(t)$ is fast in the sense that it occurs at a time $t_{B} \sim j / J$. This behavior is qualitatively captured by the first modified OTOC, with fast decay at the scrambling time and saturation at the predicted value $1 / 3$ of our phenomenological model. Interestingly, the convergence of $O_{n}(t)$ to $O(t)$ is achieved for small values, $n \gtrsim 1$. In panel (b), we represent the same quantities for a distant operator $j=7$. In this case, the dynamics of the OTOC includes a long-time slow behavior, which we attribute to the diffusive character of the operator $W(t)[22,23]$. This additional complexity of the operators (a)

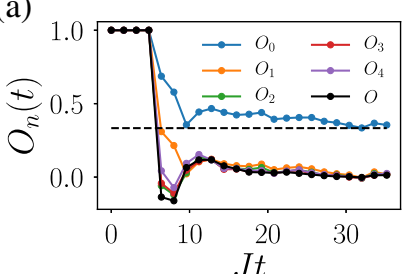

(c)

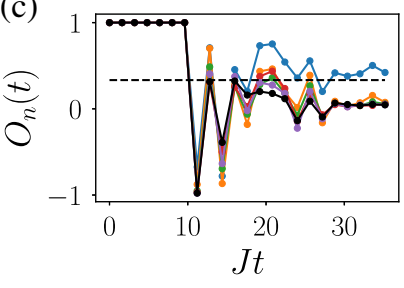

(b)
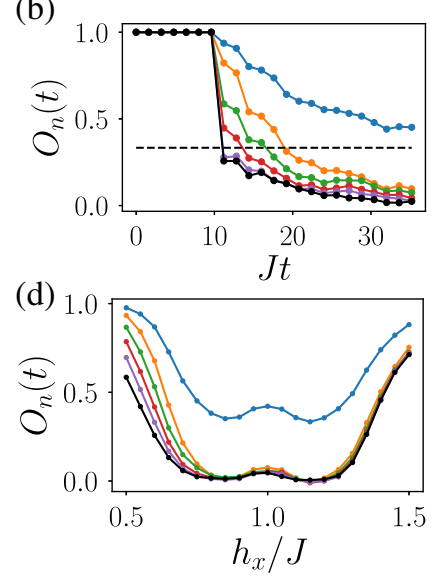

FIG. 4. $O_{n}(t)$ vs $O(t)$ for chaotic dynamics in the kicked Ising model. All plots represent for $N=8$ sites the modified OTOCs $O_{n}(t)$, for $n=0,1,2,3,4$ and the OTOC $O(t)$. The dashed line shows the predicted value $1 / 3$ for $O_{0}(t)$ for Haar scrambling. (a) For $h_{x}=0.75 \mathrm{~J}$, and $j=3$, the OTOC decay is fast and well captured by the low-order OTOCs $O_{n}(t)$. (b) For a larger $j=7$ operator position, the OTOC decay is slower, as a consequence of the diffusive broadening of $W(t)$. (c) For $j=7$, and $h_{x}=J$, the decay of $O(t)$ is fast but accompanied by strong oscillations, which are also resolved by the modified OTOCs. (d) For a fixed long time $J t=35.2$, the modified OTOCs provide good approximations to $O(t)$, in particular in the regime $h_{x} \geq 0.75 J$. Other parameters are $h_{z}=0.809 \mathrm{~J}$, and $J T=1.6$. 
[compared to ballistic spreading as in panel (a)] is captured for spatial resolutions $n \gtrsim 4$. In panel (c), we show another example of deviation from ballistic scrambling, with strong oscillations of the OTOCs, which are quantitatively captured for $n \geq 2,3$. Finally, we show in panel (d) the different regimes of scrambling as a function of the transverse field $h_{x}$ for a fixed large time $J t=35.2$. All modified OTOCs identify a region of fast scrambling around $h_{x} \sim J$. Interestingly, the modified OTOCs with $n \geq 1$ also provide an excellent approximation to $O(t)$ in a regime of slow scrambling, $h_{x} \geq 1.25 \mathrm{~J}$. Finally, for $h_{x} \leq 0.75 \mathrm{~J}$, an increased resolution is necessary to access $O(t)$. This example shows that the required resolution $n$ to access $O(t)$ up to a given error depends on the type of evolution realized by $U(t)$ and is generically small. Note that these results also suggest that the convergence properties of the series $O_{n}(t)$ can be useful to identify different regimes of scrambling.

\section{MBL dynamics with modified OTOCs}

As a second example, with the opposite type of scrambling, we consider MBL, which is the paradigmatic example of a closed quantum system that does not thermalize [58,59]. As a key signature, the decay of $O(t)$ is slow: It occurs at a characteristic time $t_{B}$ that scales exponentially with the distance $r$ between the support of $W$ and $V$ at $t=0$ $[24,26]$, which has to be contrasted to the linear increasing $t_{B} \propto r$ of chaotic systems.

To begin our analysis, we first calculate the modified OTOC $O_{0,1}(t)$ for the phenomenological $\ell$-bit model $[60,61]$ described by the Hamiltonian

$$
H=\sum_{i} h_{i}^{z} \sigma_{i}^{z}+\sum_{i<j} J_{\mathrm{R}, i j} e^{-|j-i| / \xi} \sigma_{i}^{z} \sigma_{j}^{z},
$$

with $h_{i}^{z}$ random fields, $J_{\mathrm{R}, i j}$ random interaction strengths that are taken uniformly in $\left[-J_{z}, J_{z}\right]$, and $\xi$ the localization length. Here, we only consider 2-body interaction terms, which is sufficient to show that MBL exhibits slow scrambling [24,26]. With $U(t)=e^{-i H t}, W=\sigma_{j}^{x}$, and $V=\sigma_{1}^{x}$, one finds [24]

$$
\begin{aligned}
O(t) & =\operatorname{sinc}\left(4 J_{z} e^{-r / \xi} t\right), \\
O_{0}(t) & =\frac{1+2 O(t)}{2+O(t)}, \quad O_{1}(t)=O(t),
\end{aligned}
$$

with $r=j-1$. Thus, for slow MBL scrambling, $O_{0}(t)$ is related to $O(t)$ via a simple transformation, while we obtain an exact equivalence between the one-site resolved modified OTOCs $O_{1}(t)$ and $O(t)$. In the noninteracting case $J_{z}=0$, each $\ell$ bit evolves independently, $O(t)=$ $O_{0,1}(t)=1=$ const. With interactions $J_{z}>0$, the decay of both $O(t)=O_{1}(t)$ and $O_{0}(t)$ occurs at $t_{c}=e^{r / \xi} / J_{z}$. This analytical result is shown in Fig. 5(a). Note that, similar to
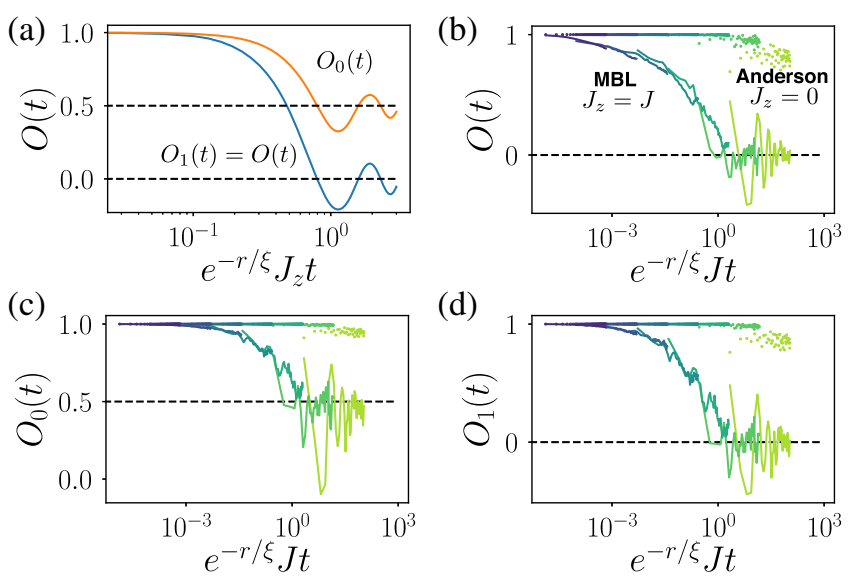

(d)

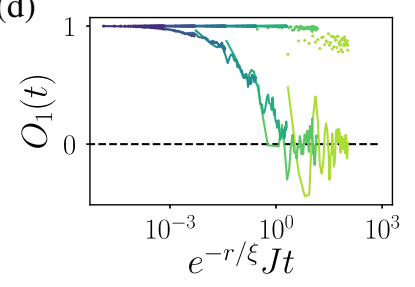

FIG. 5. MBL dynamics. (a) Values of $O(t)$ and $O_{0,1}(t)$ in the $\ell$-bit model. (b)-(d) Numerical simulations for a disordered $X X Z$ chain showing that the generic scaling of $O_{0}(t)$ matches $O(t)$, while $O(t) \approx O_{1}(t)$. We use 20 random realizations of the disorder. Green to purple curves represent increasing values of $j=2, \ldots, N$. The lines (circles) correspond to $\mathrm{MBL} J_{z}=J$ (Anderson $J_{z}=0$ ) dynamics, respectively. The $x$ axis is rescaled using a fitted localization length $\xi=2$. Other parameters: $N=8$, $\Delta=10 \mathrm{~J}$. The dashed lines represent the predictions $O(\infty)=0$ and $O_{0}(\infty)=1 / 2$.

the case of chaotic dynamics, $O(t)$ tends to zero at long times, while $O_{0}(t)$ saturates to a finite value, here $1 / 2$. These results are confirmed by our numerical simulations of a disordered $X X Z$ chain [62] shown in Figs. 5(b) and $5(\mathrm{~d})$. For the noninteracting case $J_{z}=0$, both OTOC values remain close to 1 . In the MBL phase, the decay of $O_{0,1}(t)$ exhibits the expected exponential scaling with the distance $r$, and it converges at long times to the predicted values of $1 / 2$ and 0 , respectively. As predicted by the $\ell$-bit model, the values of $O(t)$ and $O_{1}(t)$ are almost identical.

\section{Information scrambling by long-range interactions with modified OTOCs}

So far, we have considered examples where interactions are local, with analytical models supporting the statement that low-order $n$ modified OTOCs provide good approximations of $O(t)$. To conclude, we numerically study scrambling in a long-range interacting model. This situation is particularly interesting as the decay of the OTOCs is not necessarily controlled by a butterfly velocity associated with the presence of a light cone $[63,64]$.

Here, we consider a long-range $X Y$ model, realized, for instance, in trapped ion experiments [2], with timeevolution operator $U(t)=\exp \left(-i H_{\mathrm{LR}} t\right)$, and

$$
H_{\mathrm{LR}}=\sum_{j>i} \frac{J}{(j-i)^{\alpha}}\left(\sigma_{i}^{+} \sigma_{j}^{-}+\text {H.c. }\right)
$$



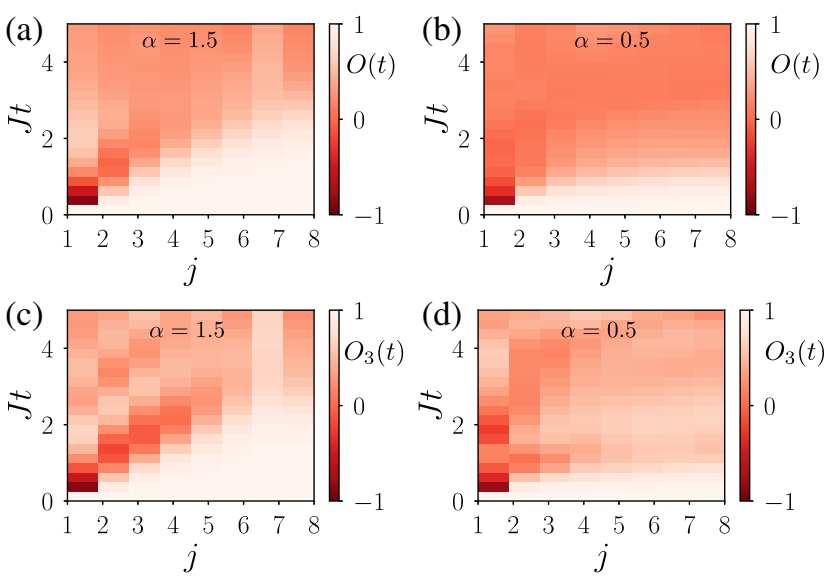

FIG. 6. Scrambling with long-range interactions. (a,b) OTOC $O(t)$ for $N=8$, and two values of $\alpha=1.5,0.5$. (c,d) Same as panels (a) and (b) for the modified OTOC $\mathrm{O}_{3}(t)$, which also distinguishes the two regimes of scrambling.

with $\alpha$ a positive number controlling the range of the interactions.

We show in Fig. 6 the space-time expansion of $O(t)$ and $O_{3}(t)$ for two different values of $\alpha$. For $\alpha=1.5$, the system satisfies Lieb-Robinson bounds [63,64], meaning that the system behaves effectively as if the interactions are local. This result is manifested by a light-cone spreading of the OTOCs. Conversely, for $\alpha=0.5$, the characteristic decay time of $O(t)$ is superlinear with respect to the operator position $j$.

In such a model with long-range interactions, the convergence of the modified OTOCs is slightly slower than for the examples shown before with nearest-neighbor interactions. However, the two types of light cones are well resolved by the modified OTOC $O_{3}(t)$. Within the light cone, $O_{3}(t)$ oscillates weakly with time. These oscillations fade out with increasing $n$.

\section{ERRORS AND IMPERFECTIONS}

We conclude our manuscript by presenting a study of errors and imperfections. From this analysis, we can draw the conclusion that OTOCs can be measured with good precision in AMO and superconducting qubit experiments with current technology, and with the total number of measurements compatible with state-of-the-art repetitions rates [10].

\section{A. Statistical errors}

Statistical errors arise in an experiment from a finite number $N_{M}$ of measurements per random unitary $u$ to access the expectation values $\langle W(t)\rangle_{u, k_{0}}$ and $\left\langle V^{\dagger} W(t) V\right\rangle_{u, k_{0}}$, and from a finite number of random unitaries $N_{u}$ used to estimate the correlation coefficients. This results in deviations $\mathcal{E}=$ $\left|[O(t)]_{e}-O(t)\right|$ between estimated and exact correlation coefficients.
The scaling of statistical errors can be explained best in terms of the phenomenological model for scrambling Eqs. (9) and (10). Accordingly, using global unitaries, the typical value of $\langle W(t)\rangle_{u, k_{0}} \sim 1 / \sqrt{2^{N}}$ is suppressed exponentially. The number of projective measurements $N_{M}$ required to access $O(t)$ up to a given error thus scales as $2^{N}$. The protocol based on local unitaries accessing $O_{n}(t)$ has a crucial advantage, provided that low resolution $n \ll N$ is sufficient to approximate $O(t)$. The typical value of the expectation values $\langle W(t)\rangle_{u, k_{s}} \sim$ $1 / \sqrt{2^{L(t)}}$ scales instead with the effective complexity $2^{L(t)}$ of the operator. Accordingly, the early-time dynamics of large chaotic systems subject to Lieb-Robinson bounds [22,23], but also the long time evolution of a MBL system, both of which correspond to small scrambling lengths $L(t) \ll N$, are accessible with a moderate number of measurements, about $2^{L(t)}$. These findings are confirmed by the numerical simulations of Fig. 7, showing the statistical error $\mathcal{E}$ for the global protocol [panel (a)] and the corresponding error $\mathcal{E}_{1}$ for the local protocol with $n=1$ [panel (b)]. Note that for both protocols, when convergence with respect to $N_{M}$ is reached, the typical statistical error is $1 / \sqrt{N_{u}}$ (consistent with the central limit theorem).
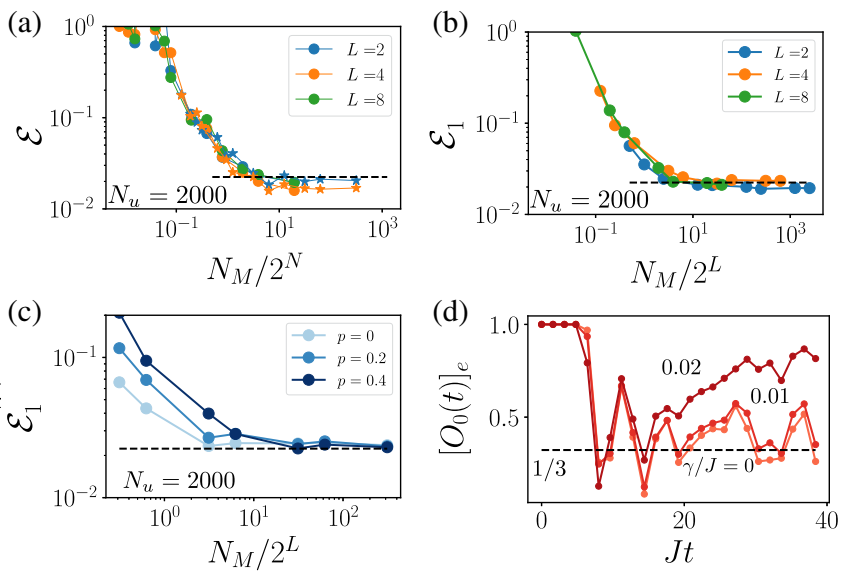

FIG. 7. Statistical errors, imperfections, and decoherence. (a,b) Statistical errors for global and local protocols in the case of the scrambling model, Eqs. (9) and (10). For $N_{M} \gg 2^{N}$ (a), resp. $N_{M} \gg 2^{L}$ (b), the errors reach a statistical plateau at $1 / \sqrt{N_{u}}$. For the simulations of the global protocols, we use the two values $N=4,8$ (circles, stars). (c) Effects of depolarization on statistical errors: This decoherence mechanism rescales the values of the observables, which implies that one should perform a slightly larger number of measurements to access $O_{1}(t)$ with a given precision. (d) Decoherence vs scrambling in the kicked Ising model. Each qubit is subject to spontaneous emission with rate $\gamma$. Parameters are $N=6, j=4, h_{x}=J, h_{z}=0.809 J, N_{M}=\infty$, $N_{u}=100$. The effect of decoherence is manifested by an increase of the correlations at long times. 


\section{B. Imperfections and decoherence}

Our two protocols have a certain robustness against various types of experimental imperfections and decoherence. First, let us consider the effect of imperfect implementations of the local random unitaries $u$, which we can parametrize with a characteristic mismatch angle $d \theta$ in the realization of spin rotations. Our error analysis presented in Appendix F 1 shows that our estimations of the OTOCs are only affected in second order in $d \theta$. The absence of a linear correction in $d \theta$ indeed illustrates a robustness mechanism inherent to protocols based on random measurements: OTOCs are estimated via an ensemble average over many random unitaries, which tends to "average out" the effect of errors from individual measurements. In particular, we expect the global protocol to have a similar robustness mechanism with respect to small errors in the preparation of global unitaries.

The two protocols are also robust against readout errors. In the case of decoherence, depolarization noise only rescales the values of the measurements of $W$ while leaving statistical correlations unaltered [see Appendix F2 and Fig. 7(c)]. For other sources of decoherence, the values of the correlations can be affected but in a way that can be clearly distinguished from unitary scrambling. While scrambling generically leads to a decay of statistical correlations, decoherence increases correlations. This behavior, which is the opposite of the case of protocols based on the reversal of time evolution $[39,43,44]$, can be understood by noting that, for a Markovian dissipative evolution, the distance between two different states always decreases with time [65]. This case is illustrated in Fig. 7(d) for the estimation of the modified OTOC $\left[O_{0}(t)\right]_{e}$.

\section{CONCLUSION AND OUTLOOK}

In the present work, we provide novel protocols to measure OTOCs for spin models, based on statistical correlations between measurement outcomes obtained from random initial states from both global and local random unitaries. These protocols can be implemented in state-ofthe-art quantum simulation experiments on various physical platforms, in particular, in Rydberg atoms, trapped ions, or superconducting qubits, which provide high repetition rates. The paradigm of extracting nonstandard correlation functions of quantum many-body systems from statistical correlations points to several interesting future developments, including extensions to measure modified OTOCs for Hubbard models [45].

While we have focused on second-order statistical correlations based on cross-correlating two random measurements, higher-order moments can also be used-at the price of higher requirements w.r.t. statistical errors-to access higher-order OTOCs [33] or OTOCs of arbitrary states $\rho$. In particular, we show in Appendix G 1 that the protocol can be adapted to access "symmetrized" OTOCs of arbitrary states, in particular, of thermal and ground states. This method could be used to extract the crucial temperature dependence of the Lyapunov exponents in models of high-energy physics such as the Sachdev-YeKitaev models [27-32].

\section{ACKNOWLEDGMENTS}

We thank M. Heyl, E. Altman, M. Dalmonte, T. Schuster, C. Roos, C. Maier, T. Brydges, and M. Joshi for discussions and comments on the manuscript. Work in Innsbruck was supported by the ERC Synergy Grant UQUAM and the Austrian Science Fund (FWF SFB FoQus Project No. F4016- N23). N. Y. Y. acknowledges support from the DOE under Contract No. PHCOMPHEP-KA24 and the Office of Advanced Scientific Computing Research, Quantum Algorithm Teams Program. Numerical simulations were realized with QuTiP [66].

\section{APPENDIX A: CORRESPONDENCE BETWEEN STATISTICAL CORRELATIONS AND OTOCS WITH LOCAL UNITARIES}

In this section, we prove the relation between statistical correlations and OTOCs for the second protocol with local random unitaries $u=u_{1} \otimes \cdots \otimes u_{N}$, where each $u_{i}$ is sampled independently from $\operatorname{CUE}(d), d$ being the local Hilbert space dimension $(d=2$ for the spin- $1 / 2$ we consider). Here, we extend our diagrammatic approach by introducing a matrix-product-operator (MPO) [67] representation for the operators $W(t)$ and $V$. This extension is shown in Fig. 8: Each (blue) physical index is contracted following the 2-design rule shown in panel (a), while the (green) bond links remain unchanged. To simplify the derivation of the proof, we rewrite $O_{n}(t)$ as

$$
O_{n}(t) \equiv \frac{\overline{\langle W(t)\rangle_{u, n}\left\langle V^{\dagger} W(t) V\right\rangle_{u, k_{0}}}}{\overline{\langle W(t)\rangle_{u, n}\langle W(t)\rangle_{u, k_{0}}}}
$$

with $\quad\langle W(t)\rangle_{u, n} \equiv \sum_{k_{s} \in E_{n}}(-1 / 2)^{d\left[k_{s}, k_{0}\right]}\langle W(t)\rangle_{u, k_{s}}=$ $\operatorname{Tr}\left[r_{n} W(t)\right]$, with the operator $r_{n}=r_{1, n} \otimes \ldots \otimes r_{1, N}$ written as a tensor product with $r_{i, n}=\left|k_{0}^{(i)}\right\rangle\left\langle k_{0}^{(i)}\right|-$ $1 / 2\left(\sigma_{i}^{x}\left|k_{0}^{(i)}\right\rangle\left\langle k_{0}^{(i)}\right| \sigma_{i}^{x}\right) \delta_{i \leq n}$. The operator $r_{n}$ gathers all the information about the initial states $\left|k_{s}\right\rangle \in E_{n}$ and the corresponding chosen weights $c_{k_{s}}=(-1 / 2)^{d\left[k_{0}, k_{s}\right]}$. Finally, we also use the tensor product decomposition $\rho_{0}=\rho_{1} \otimes \ldots \otimes \rho_{N}$, with $\rho_{i}=\left|k_{0}^{(i)}\right\rangle\left\langle k_{0}^{(i)}\right|$.

We can now prove graphically, in Fig. 8,

$$
\begin{aligned}
& \overline{\langle W(t)\rangle_{u, n}\langle V W(t) V\rangle_{u, k_{0}}} \\
& =\frac{1}{3^{N}}\left(\frac{3}{4}\right)^{n}\left(\frac{1}{2}\right)^{N-n} \sum_{\tau \in \mathcal{E}_{n}^{(\mathrm{L})}} \operatorname{Tr}(\tau W(t) \otimes V W(t) V),
\end{aligned}
$$




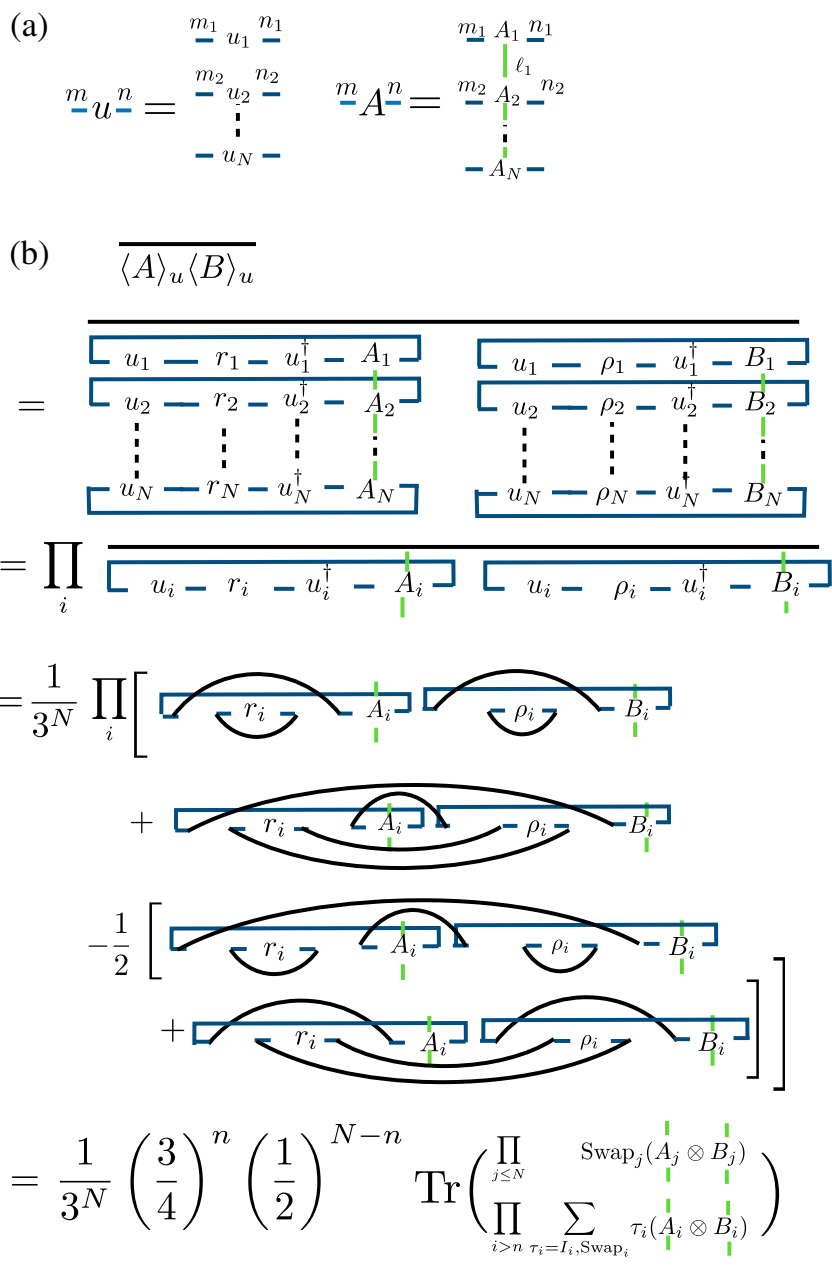

FIG. 8. Identities for our protocol with local unitaries. (a) MPO representation of multisite indexed operators. (b) Correlations between two measurements with $A=W(t), B=V^{\dagger} W(t) V$.

where the index $n$ is omitted in the graphics. The ensemble $\mathcal{E}_{n}^{(\mathrm{L})}$ consists of all the $2^{N-n}$ permutations of the form $\tau=\prod_{j \geq n} \operatorname{swap}_{j} \prod_{i>n} \tau_{i}, \quad \tau_{i}=I_{i}, \operatorname{swap}_{i}, \quad$ with the local swap operator $\operatorname{swap}_{i}\left|k_{i}\right\rangle \otimes\left|k_{i}^{\prime}\right\rangle=\left|k_{i}^{\prime}\right\rangle \otimes\left|k_{i}\right\rangle$. This case leads directly to the desired equality

$$
\begin{aligned}
O_{n}(t) & =\frac{\sum_{\tau \in \mathcal{E}_{n}^{(\mathrm{L})}} \operatorname{Tr}\left\{\tau\left[W(t) \otimes V^{\dagger} W(t) V\right]\right\}}{\sum_{\tau \in \mathcal{E}_{n}^{(\mathrm{L})}} \operatorname{Tr}\{\tau[W(t) \otimes W(t)]\}} \\
& =\frac{\sum_{A,\{1 \ldots n\} \subseteq A} \operatorname{Tr}_{A}\left(W_{A}(t)\left[V^{\dagger} W(t) V\right]_{A}\right)}{\sum_{A,\{1 \ldots n\} \subseteq A} \operatorname{Tr}_{A}\left[W_{A}^{2}(t)\right]} .
\end{aligned}
$$

In particular, for $n=N$, we obtain $O_{N}(t)=O(t)$.

\section{APPENDIX B: OTOCS FOR HAAR SCRAMBLING}

We now prove Eqs. (9) and (10) of the main text. The case $j>L(t)$ is straightforward due to the commutativity of $V$ and $W$. For $j \leq L(t)$, the equality written for the (a)

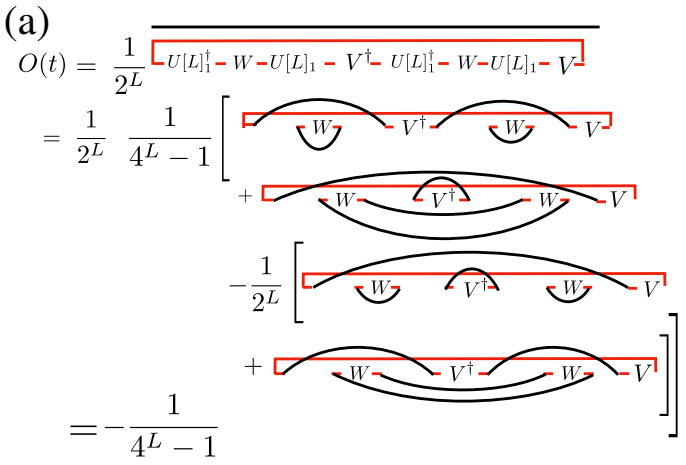

(b) $\overline{\langle W(t)\rangle_{u}\left\langle V^{\dagger} W(t) V\right\rangle_{u}}$

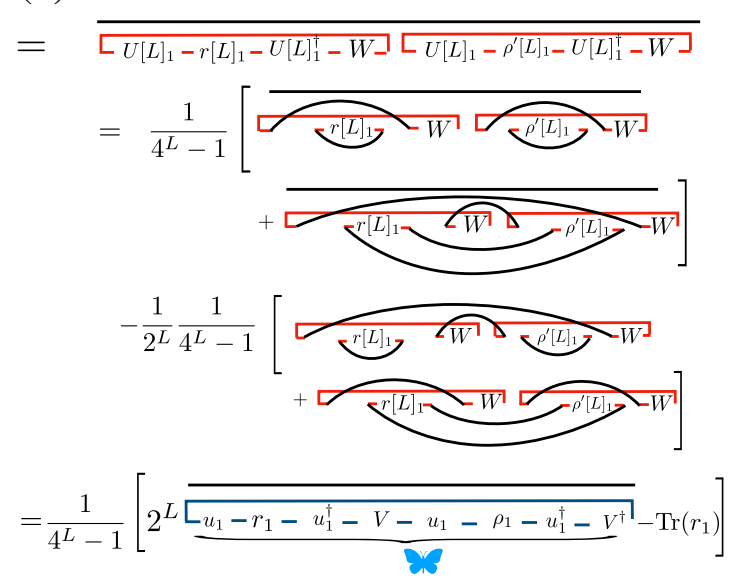

(c)

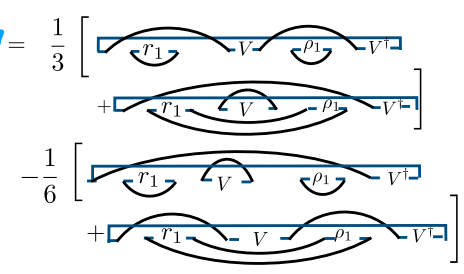

FIG. 9. Proof of Eq. (9) using a diagrammatic approach. In panels (a) and (b), the ensemble average is performed over the unitaries $U$ leading to the contractions of the red indices. In panel (c), the ensemble average is taken with respect to the unitaries $u_{1}$ with the contracted indices shown in blue.

OTOC $O(t)$ follows directly from the 2-design identities [cf. Fig. 9(a)] and can also be found in Ref. [33].

For the modified OTOCs, we use the mapping to statistical correlations to calculate the values corresponding to Haar scrambling. We show in Figs. 9(b) and 9(c) the evaluation of $\overline{\langle W(t)\rangle_{u, n=0,1}\left\langle V^{\dagger} W(t) V\right\rangle_{u, k_{0}}}$, which can be adapted to derive $\overline{\langle W(t)\rangle_{u, n}\langle W(t)\rangle_{u, k_{0}}}$ by replacing $V$ by the identity matrix. Since $u$ is a product of local random unitaries, we use the decomposition $X=X[L]_{1} \otimes, \ldots$, $X[L]_{1}=\bigotimes_{i \leq L} u_{i} X_{i} u_{i}^{\dagger}$, for $X=r, \rho$. We also use the notation $\rho^{\prime}[L]_{1}=V \rho[L]_{1} V^{\dagger}$. This method proves the desired identities

$$
O_{0}(t)=\frac{2^{L} / 3-1}{2^{L}-1}, \quad O_{1}(t)=\frac{-1}{2^{L+1}-1} .
$$




\section{APPENDIX C: OTOCs FOR MANY-BODY LOCALIZATION}

To describe analytically the behavior of OTOCs in the MBL phase [24,26], we consider the $\ell$-bit model with time evolution $U(t)=\exp (-i H t)$ described by the Hamiltonian

$$
H=\sum_{i} h_{i}^{z} \sigma_{i}^{z}+\sum_{i<j} J_{i, j} \sigma_{i}^{z} \sigma_{j}^{z}
$$

where $h_{i}^{z}$ are random fields that, as we show below, have no effect on the OTOC; $J_{i j}=J_{\mathrm{R}, i j} \exp (-|j-i| / \xi)$, where $J_{\mathrm{R}, i j}$ is a random interaction amplitude with probability distribution $f\left(J_{\mathrm{R}, i j}\right)$, which we assume to be uniform in $\left[-J_{z}, J_{z}\right]$, and $\xi$ is the localization length. We study the behavior of the OTOCs for the operators $W=\sigma_{j}^{x}$ and $V=\sigma_{1}^{x}$. In the Anderson case $J_{z}=0$, each $\ell$ bit evolves independently, and we have $O(t)=O_{n}(t)=1$.

\section{Single disorder realization}

We now address the general MBL case $J_{z}>0$ and first consider a single disorder realization of the interaction matrix $\left(J_{i j}\right)$, writing the Heisenberg operators as

$$
\begin{aligned}
W(t) & =\sigma_{j}^{x} e^{-2 i t h_{j}^{z} \sigma_{j}^{z}} e^{-2 i t \sum_{i \neq j} J_{i j} \sigma_{i}^{z} \sigma_{j}^{z}}, \\
V^{\dagger} W(t) V & =\sigma_{j}^{x} e^{-2 i t h_{j}^{z} \sigma_{j}^{z}} e^{-2 i t \sum_{i \neq j} J_{i j} c_{i} \sigma_{i}^{z} \sigma_{j}^{z}},
\end{aligned}
$$

with $c_{i \neq 1}=1, c_{1}=-1$. We then obtain

$$
O(t)=\frac{1}{2^{N}} \operatorname{Tr}\left[\exp \left(-4 i t J_{1 j} \sigma_{1}^{z} \sigma_{j}^{z}\right)\right]=\cos \left(4 J_{1 j} t\right),
$$

as already shown in Refs. [24,26]. We now evaluate $O_{n=0,1}(t)$ using the first line given in Eq. (A3). We first perform the trace operation with respect to the site $j$,

$$
\begin{gathered}
\operatorname{Tr}_{j}\left\{\left(I_{j}+\operatorname{swap}_{j}\right)[W(t) \otimes W(t)]\right\} \\
=2 \cos \left(2 t \sum_{i \neq j} J_{i j}\left(\sigma_{i}^{z}-\tilde{\sigma}_{i}^{z}\right)\right), \\
\operatorname{Tr}_{j}\left[\left(I_{j}+\operatorname{swap}_{j}\right)\left(W(t) \otimes V^{\dagger} W(t) V\right)\right] \\
=2 \cos \left(2 t \sum_{i \neq j} J_{i j}\left(\sigma_{i}^{z}-c_{i} \tilde{\sigma}_{i}^{z}\right)\right),
\end{gathered}
$$

with $\tilde{\sigma}_{i}^{\beta}(\beta=x, y, z)$ the set of Pauli matrices in the "copy" Hilbert space $\mathcal{H}_{i}$ associated with site $i$. We can then calculate the trace over the remaining sites, for instance,

$$
\begin{aligned}
& \operatorname{Tr}_{k}\left[\left(I_{k}+\operatorname{swap}_{k}\right) \cos \left(2 t \sum_{i \neq j} J_{i j}\left(\sigma_{i}^{z}-\tilde{\sigma}_{i}^{z}\right)\right)\right] \\
& =\left[4 \cos \left(2 t J_{k j}\right)^{2}+2\right] \cos \left(2 t \sum_{i \neq k, j} J_{i j}\left(\sigma_{i}^{z}-\tilde{\sigma}_{i}^{z}\right)\right), \\
& \operatorname{Tr}_{k}\left[\left(I_{k}+\operatorname{swap}_{k}\right) \cos \left(2 t \sum_{i \neq j} J_{i j}\left(\sigma_{i}^{z}-c_{i} \tilde{\sigma}_{i}^{z}\right)\right)\right] \\
& =\left[4 \cos \left(2 t J_{k j}\right)^{2}+2 \cos \left(2\left(1-c_{k}\right) J_{k j} t\right)\right] \\
& \quad \times \cos \left(2 t \sum_{i \neq k, j} J_{i j}\left(\sigma_{i}^{z}-\tilde{\sigma}_{i}^{z}\right)\right) .
\end{aligned}
$$

All the factors that enter in the numerator and in the denominator in Eq. (A3) are identical, except for the position $k=1$ of the $V$ operator. This case leads to

$$
\begin{aligned}
& O_{0}(t)=\frac{4 \cos \left(2 J_{1 j} t\right)^{2}+2 \cos \left(4 J_{1 j} t\right)}{4 \cos \left(2 J_{1 j} t\right)^{2}+2}=\frac{2 \cos \left(4 J_{1 j} t\right)+1}{\cos \left(4 J_{1 j} t\right)+2}, \\
& O_{1}(t)=\cos \left(4 J_{1 j} t\right) .
\end{aligned}
$$

\section{Averaged OTOCs}

Considering a distribution of the random realizations of $J_{i j}, O_{n=0,1}(t)$ is now obtained from Eq. (A3), where the numerator and the denominator are replaced by their ensemble average. Repeating the above derivation, replacing each cosine contribution by the average

$$
\cos \left(\alpha J_{i j} t\right) \rightarrow \int_{-J_{z}}^{J_{z}} d J_{\mathrm{R}, i j} \cos \left(\alpha J_{\mathrm{R}, i j} e^{-|j-i| / \xi} t\right)
$$

we obtain Eq. (13).

\section{Simulations of the disordered $X X Z$ model}

To describe the OTOCs in the MBL dynamics [24], we consider the $X X Z$ model

$H=\sum_{i}\left[J\left(\sigma_{i}^{+} \sigma_{i+1}^{-}+\sigma_{i}^{-} \sigma_{i+1}^{+}\right)+J_{z} \sigma_{i}^{z} \sigma_{i+1}^{z}\right]+\sum_{i} h_{z}^{i} \sigma_{i}^{z}$,

with $h_{z}^{i}$ sampled from a uniform distribution $[-\Delta, \Delta]$.

\section{APPENDIX D: NUMERICAL SIMULATION WITH DECOHERENCE}

To study the competition between scrambling dynamics and decoherence, we consider the kicked Ising model. The dynamics is calculated from the Lindblad master equation 


$$
\begin{aligned}
& \dot{\rho}(t)=-i[H(t), \rho(t)]+\gamma \sum_{i} \mathcal{L}\left[\sigma_{i}^{-}\right][\rho(t)], \\
& H\left(t \in\left[(n-1) T, n T-\frac{T}{2}\right]\right)=h_{x} \sum_{i} \sigma_{i}^{x}, \\
& H\left(t \in\left[n T-\frac{T}{2}, n T\right]\right)=J \sum_{i} \sigma_{i}^{z} \sigma_{i+1}^{z}+\sum_{i} h_{z} \sigma_{i}^{z}, \\
& \mathcal{L}\left[\sigma_{i}^{-}\right](\rho)=\frac{1}{2}\left[2 \sigma_{i}^{-} \rho \sigma_{i}^{+}-\rho \sigma_{i}^{+} \sigma_{i}^{-}-\sigma_{i}^{+} \sigma_{i}^{-} \rho\right],
\end{aligned}
$$

with the initial condition $\rho(0)=\rho_{0}$, and $\gamma$ the spontaneous emission decay rate (identical for each qubit).

\section{APPENDIX E: NUMERICAL STUDY OF STATISTICAL ERRORS}

Here, we present complementary data to Fig. 7, showing the scaling of statistical errors in our protocol with global and local unitaries. The results are shown in Fig. 10. The data confirm that statistical errors for $N_{M} \rightarrow \infty$ decrease as $1 / \sqrt{N_{u}}$ with a growing number of applied random unitaries $N_{u}$, independently of $L(t)$ and $N$ [panels (a)-(c)]. Panel (d) shows the scaling of statistical errors with respect to $N_{M}$ for the first modified OTOC.

\section{APPENDIX F: ROBUSTNESS OF THE PROTOCOLS}

In this section, we give three examples showing how random measurements are robust against different kinds of perturbations. In each case, we consider the protocol with local unitaries.
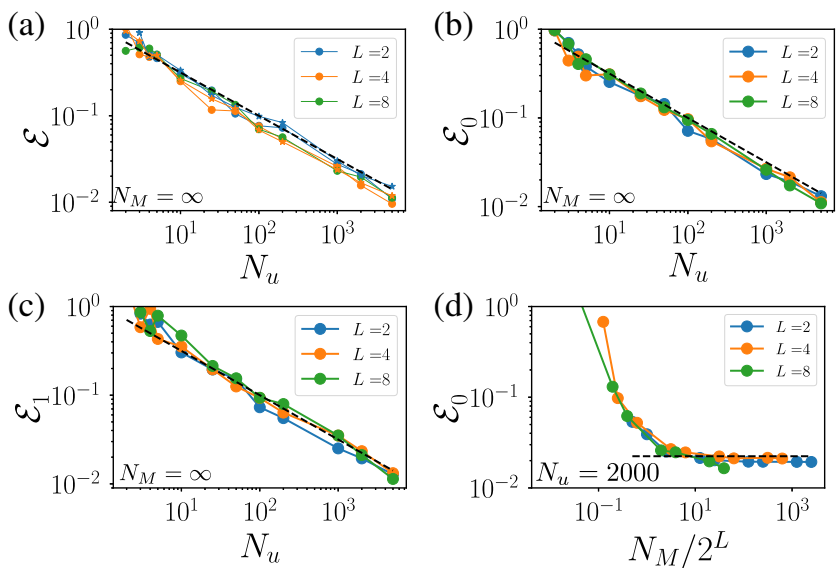

FIG. 10. Additional simulations on statistical errors. (a) Scalings of the error $\mathcal{E}$ in the estimation of $O(t)$ as a function of $N_{u}$ with global unitaries, and $N_{M} \rightarrow \infty$. We use the two values $N=4,8$ (circles, stars). (b,c) Same as panel (a) for local unitaries estimating $O_{0,1}(t)$ (here, the simulations are independent of $N$ ). (d) Statistical errors for the estimation of $O_{0}(t)$ as a function of $N_{M}$, and $N_{u}=2000$. In all panels, the dashed lines represent $1 / \sqrt{N_{u}}$.

\section{Limited reproducibility of generated random unitaries}

First, we analyze the robustness of our protocol with respect to imperfections in the generation of random unitaries. Specifically, we assume that $\langle W(t)\rangle_{u, k_{s}}$ is obtained from a random unitary matrix $u=u_{1} \otimes \cdots u_{N}$, with $u_{i} \in \operatorname{CUE}(2)$, while the second measurement $\left\langle V^{\dagger} W(t) V\right\rangle_{u^{\prime}, k_{0}}$ is obtained from a slightly different unitary $u^{\prime}=u_{1}^{\prime} \otimes \cdots u_{N}^{\prime}$, which we write as

$$
u_{i}^{\prime}=R_{i z}\left(\theta_{i 1}\right) R_{i y}\left(\theta_{i 2}\right) R_{i z}\left(\theta_{i 3}\right) u_{i} .
$$

Here, $R_{i \gamma}$ denotes single qubit rotations for spin $i$ along the $\gamma$ axis, and $\theta_{\text {in }}$ are assumed to be random angles drawn uniformly in $[-\theta, \theta]$, which represent the unwanted mismatch between the unitaries $u$ and $u^{\prime}$.

\section{a. Analytical understanding of the robustness of the protocol}

To show that our protocol is robust against such imperfections, we calculate the estimator of the OTOC, obtained from

$$
\tilde{O}_{n}(t)=\frac{\sum_{k_{s} \in E_{n}} c_{k_{s}} \overline{\langle W(t)\rangle_{u, k_{s}}\left\langle V^{\dagger} W(t) V\right\rangle_{u^{\prime}, k_{0}}}}{\sum_{k_{s} \in E_{n}} c_{k_{s}} \overline{\langle W(t)\rangle_{u, k_{s}}\langle W(t)\rangle_{u, k_{0}}}} .
$$

To first order $\theta_{\text {in }}$, we can write $u^{\prime}=u+\sum_{i, n} \theta_{\text {in }} A_{\text {in }}$, with $A_{\text {in }}=R_{\text {in }}^{\prime}(0) u$. This leads to

$$
\begin{aligned}
\overline{\langle W(t)\rangle_{u, k_{s}}\left\langle V^{\dagger} W(t) V\right\rangle_{u^{\prime}, k_{0}}} \\
=\overline{\langle W(t)\rangle_{u, k_{s}}\left\langle V^{\dagger} W(t) V\right\rangle_{u, k_{0}}} \\
\quad+\sum_{i, n} \overline{\theta_{\text {in }}} \overline{\langle W(t)\rangle_{u, k_{s}} \operatorname{Tr}\left(\left[u \rho_{0} A_{\mathrm{in}}^{\dagger}+A_{\mathrm{in}} \rho_{0} u^{\dagger}\right] V^{\dagger} W(t) V\right),}
\end{aligned}
$$

where the second term vanishes because of $\overline{\theta_{\text {in }}}=0$. This implies that $\tilde{O}_{n}(t)=O_{n}(t)$. Our protocol is thus robust to first order in $\theta$ against imperfections of the generated unitaries. As shown below, the quadratic contribution scales linearly with the characteristic size $L(t)$ of the operator $W(t)$.

\section{b. Numerical example}

We now consider a numerical example for the model of scrambling presented in Sec. III B and the estimation of the first modified OTOC $n=0$. To assess the robustness of statistical correlations, we consider $j>L(t)$, so that the exact OTOC is $O_{0}(t)=1$, and imperfect generated unitaries as written above.

The numerical results are shown in Fig. 11 and confirm our analytical treatment: There is no linear correction in $\theta$ in the error $\mathcal{E}$ of the estimated OTOC. However, the error 


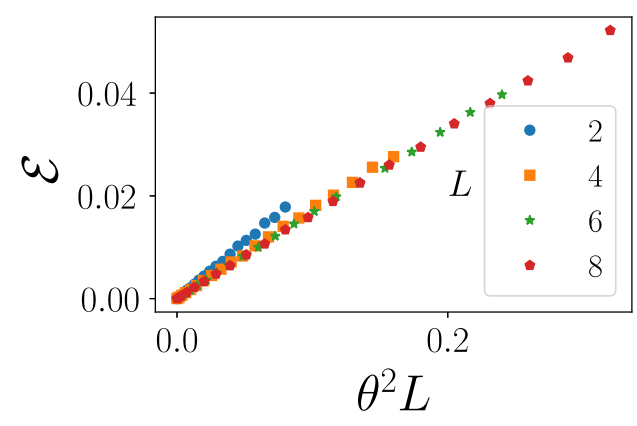

FIG. 11. Robustness of the protocol with respect to a mismatch between generated random unitaries in the estimation of the first modified OTOC. The error $\mathcal{E}$ is plotted as a function of the scaling parameter $\theta^{2} L$, for $L=4,6,8$ and $0<\theta<0.2$ Note that for all data points, the error is below $6 \%$.

scales as $\theta^{2} L$, showing that the quadratic contribution does not vanish.

\section{Robustness against the depolarizing channel}

In the presence of depolarizing noise [39], the realized quantum state can be written as

$$
\rho_{f}=(1-p) U_{\mathrm{tot}}(t) \rho_{0} U_{\mathrm{tot}}^{\dagger}(t)+\frac{p I}{d^{N}},
$$

with $0<p<1$ the depolarization probability, $I$ the identity matrix, and $U_{\text {tot }}(t)$ the desired time-evolution unitary operator. This case translates to the measurement outcomes [for the sequence shown in Fig. 1 with $U_{\text {tot }}(t)=U(t) u$ (a) and $\left.U_{\mathrm{tot}}(t)=U(t) V u(\mathrm{~b})\right]$

$$
\begin{aligned}
\langle W(t)\rangle_{u, k_{s}}^{\prime} & =(1-p)\langle W(t)\rangle_{u, k_{s}}, \\
\left\langle V^{\dagger} W(t) V\right\rangle_{u, k_{0}}^{\prime} & =(1-p)\left\langle V^{\dagger} W(t) V\right\rangle_{u, k_{0}},
\end{aligned}
$$

where we use the fact that $W(t)$ is traceless. Hence, the two measurements are simply rescaled, and the statistical correlations are not affected by the depolarizing channel. This case is illustrated in Fig. 7(d), where the numerical simulation [for the same model and parameters as in Figs. 7(a)-7(c)] shows that the effect of depolarization can be completely eliminated by performing a sufficient number of projective measurements $N_{M}$.

\section{Robustness against readout errors}

We now show that our protocols are also robust against readout errors. Here, we consider, for concreteness, $W=\sigma_{j}^{z}$. For each random unitary $u$, and initial state $k_{s}$ $\left(k_{0}\right)$, we assume that each measurement of the operator is estimated as

$$
\begin{aligned}
\langle W(t)\rangle_{u, k_{s}}^{\text {est }} & =2 P^{\text {est }}\left(t, \uparrow, u\left|k_{s}\right\rangle\right)-1, \\
\langle V W(t) V\rangle_{u, k_{0}}^{\text {est }} & =2 P^{\text {est }}\left(t, \uparrow, u V\left|k_{0}\right\rangle\right)-1,
\end{aligned}
$$

where $P^{\text {est }}(t, \uparrow,|k\rangle)$ is the estimated probability to detect spin $j$ in state $\uparrow$ after time evolution from the state $|k\rangle$. We now consider that the estimated probabilities $P^{\text {est }}(t, \uparrow,|k\rangle)$ are built from a sequence of $N_{M}$ measurements,

$$
P^{\text {est }}(t, \uparrow,|k\rangle)=\frac{1}{N_{M}} \sum_{i} X_{i},
$$

with $X_{i}$ the measurement outcomes obtained with error probability $x$ :

$$
\begin{aligned}
\operatorname{Prob}\left(X_{i}=1\right) & =(1-x) P(t, \uparrow,|k\rangle)+x P(t, \downarrow,|k\rangle) \\
& =(1-2 x) P(t, \uparrow,|k\rangle)+x .
\end{aligned}
$$

In the limit of an infinite number of measurements $\left(N_{M} \rightarrow\right.$ $\infty)$, we obtain $P^{\text {est }}(t, \uparrow,|k\rangle)=\operatorname{Prob}\left(X_{i}=1\right)$, and thus

$$
\begin{aligned}
\langle W(t)\rangle_{u, k_{s}}^{\text {est }} & =(1-2 x)\langle W(t)\rangle_{u, k_{s}}, \\
\langle V W(t) V\rangle_{u, k_{0}}^{\text {est }} & =(1-2 x)\langle V W(t) V\rangle_{u, k_{0}}^{\text {est }} .
\end{aligned}
$$

Accordingly, as in the case of the depolarizing channel, readout errors simply rescale the value of the observables; i.e., the estimation of $O_{n}(t)$ is not affected by readout errors.

\section{APPENDIX G: ACCESSING OTOCS FOR NONINFINITE-TEMPERATURE STATES}

In this section, we show how to extend our protocol beyond the case of infinite temperature $\rho=I / \mathcal{N}_{\mathcal{H}}$.

\section{Thermal OTOCs from global unitaries}

First, we show how our protocol can be extended to include finite-temperature corrections to $O(t)$. To this end, we employ a high-temperature expansion of the thermal density matrix $\rho_{\beta}=\exp (-\beta H) / Z$, with $H$ being the many-body Hamiltonian of the system of interest $Z=$ $\operatorname{Tr}[\exp (-\beta H)]$ and $\beta$ the inverse temperature. Specifically, instead of considering the canonical finite-temperature OTOC

$$
O\left[\rho_{\beta}\right](t)=\operatorname{Tr}\left(\rho_{\beta} W(t) V W(t) V\right),
$$

with $\rho_{\beta}$ a thermal density matrix, we consider a symmetrized variant, introduced in Ref. [31],

$$
O_{S}\left[\rho_{\beta}\right](t)=\operatorname{Tr}\left(\rho_{\beta}^{1 / 4} W(t) \rho_{\beta}^{1 / 4} V \rho_{\beta}^{1 / 4} W(t) \rho_{\beta}^{1 / 4} V\right) .
$$

Here, and in the following, we assume $W(t)$ to be Hermitian, $V$ to be Hermitian and unitary, and all operators $W, V, H$ to be traceless (which is the case for the spin models considered in the main text). We employ a hightemperature expansion of $\rho_{\beta} \sim(I-\beta H) / \mathcal{N}_{\mathcal{H}}+\mathcal{O}\left(\beta^{2}\right)$. To first order in $\beta$, we find 


$$
\begin{aligned}
O_{S}\left[\rho_{\beta}\right](t)= & O(t)-\frac{\beta}{2 \mathcal{N}_{\mathcal{H}}}\{\operatorname{Tr}[H W(t) V W(t) V] \\
& +\operatorname{Tr}[H V W(t) V W(t)]\}+\mathcal{O}\left(\beta^{2}\right) .
\end{aligned}
$$

We now introduce the statistical correlations

$$
\tilde{C}(t)=\overline{\langle W(t)\rangle_{u, k_{0}}\langle V W(t) V\rangle_{u, k_{0}}\langle H\rangle_{u, k_{0}}},
$$

with $u$ global random unitaries of the CUE and $\langle A\rangle_{u, k_{0}}=$ $\left\langle\psi_{u}|A| \psi_{u}\right\rangle$. Using the 3-design properties [52] of the CUE, we obtain

$$
\tilde{C}(t)=c^{\prime} \sum_{\tau \in S_{3}} \operatorname{Tr}(\tau[W(t) \otimes V W(t) V \otimes H]),
$$

with $c^{\prime}=\left[\mathcal{N}_{\mathcal{H}}\left(\mathcal{N}_{\mathcal{H}}+1\right)\left(\mathcal{N}_{\mathcal{H}}+2\right)\right]^{-1}$. This result can be rewritten as

$$
\tilde{C}(t)=c^{\prime}\{\operatorname{Tr}[H W(t) V W(t) V]+\operatorname{Tr}[H V W(t) V W(t)]\} .
$$

Using Eqs. (G3) and (G4), and $O(t)=\tilde{O}^{(\mathrm{G})}(t)$, we obtain

$$
O_{S}\left[\rho_{\beta}\right](t)=O(t)-\frac{\beta}{2 c^{\prime} \mathcal{N}_{\mathcal{H}}} \tilde{C}(t),
$$

which shows that $O_{S}\left[\rho_{\beta}\right](t)$ can be accessed by separately measuring (i) the $T=\infty$ value $O(t)$ as described in the main text and (ii) the additional correlations $\tilde{C}(t)$. This result shows that thermal OTOCs are experimentally accessible by measuring statistical correlations. The additional requirement compared to the measurement of $O(t)$ is the measurement of the energy $\langle H\rangle_{u, k_{0}}$ of random initial states.

\section{OTOCs for arbitrary states from global unitaries}

We now generalize the previous approach and show that we can access, for an arbitrary state $\rho$ (thermal or not), another symmetric variant of OTOCs,

$$
O_{S^{\prime}}[\rho](t)=\frac{1}{2}\{\operatorname{Tr}[\rho W(t) V W(t) V]+\operatorname{Tr}[W(t) \rho V W(t) V]\}
$$

Note that for thermal states $\rho=\rho_{\beta}, O_{S^{\prime}}\left[\rho_{\beta}\right](t)$ and $O_{S}\left[\rho_{\beta}\right](t)$ coincide to first order in $\beta$. Also, when $V$ and $\rho$ commute, this variant is equivalent to the standard OTOC, i.e., $O_{S^{\prime}}[\rho](t)=O[\rho](t)$. Now, we introduce the statistical correlations

$$
\tilde{C}^{\prime}(t)=\overline{\langle W(t)\rangle_{u, k_{0}}\langle V W(t) V\rangle_{u, k_{0}}\langle\rho\rangle_{u, k_{0}}},
$$

with the same notations as in Appendix G 1. Note that the last term $\langle\rho\rangle_{u, k_{0}}$ simply consists in measuring the probability that the state $\rho$ is in the random state $\left|\psi_{u}\right\rangle$. We then obtain, using 3-design properties,

$$
\tilde{C}^{\prime}(t)=c^{\prime} \sum_{\tau \in S_{3}} \operatorname{Tr}(\tau[W(t) \otimes V W(t) V \otimes \rho])
$$

which corresponds to a sum of $O_{S^{\prime}}[\rho](t)$ and of lower-order terms that can be measured independently (such as the infinite temperature OTOC). Thus, $O_{S^{\prime}}[\rho](t)$ can be measured via statistical correlations.

[1] C. Gross and I. Bloch, Quantum Simulations with Ultracold Atoms in Optical Lattices, Science 357, 995 (2017).

[2] R. Blatt and C. F. Roos, Quantum Simulations with Trapped Ions, Nat. Phys. 8, 277 (2012).

[3] A. Browaeys, D. Barredo, and T. Lahaye, Experimental Investigations of Dipole-Dipole Interactions between a Few Rydberg Atoms, J. Phys. B 49, 152001 (2016).

[4] J. M. Gambetta, J. M. Chow, and M. Steffen, Building Logical Qubits in a Superconducting Quantum Computing System, npj Quantum Inf. 3, 2 (2017).

[5] D. Greif, M. F. Parsons, A. Mazurenko, C. S. Chiu, S. Blatt, F. Huber, G. Ji, and M. Greiner, Site-Resolved Imaging of a Fermionic Mott Insulator, Science 351, 953 (2016).

[6] L. W. Cheuk, M. A. Nichols, K. R. Lawrence, M. Okan, H. Zhang, E. Khatami, N. Trivedi, T. Paiva, M. Rigol, and M. W. Zwierlein, Observation of Spatial Charge and Spin Correlations in the 2D Fermi-Hubbard Model, Science 353, 1260 (2016).

[7] M. Boll, T. A. Hilker, G. Salomon, A. Omran, J. Nespolo, L. Pollet, I. Bloch, and C. Gross, Spin- and Density-Resolved Microscopy of Antiferromagnetic Correlations in FermiHubbard Chains, Science 353, 1257 (2016).

[8] J.-y. Choi, S. Hild, J. Zeiher, P. Schauss, A. Rubio-Abadal, T. Yefsah, V. Khemani, D. A. Huse, I. Bloch, and C. Gross, Exploring the Many-Body Localization Transition in Two Dimensions, Science 352, 1547 (2016).

[9] J. Zhang, G. Pagano, P. W. Hess, A. Kyprianidis, P. Becker, H. Kaplan, A. V. Gorshkov, Z.-X. Gong, and C. Monroe, Observation of a Many-Body Dynamical Phase Transition with a 53-Qubit Quantum Simulator, Nature (London) 551, 601 (2017).

[10] T. Brydges, A. Elben, P. Jurcevic, B. Vermersch, C. Maier, B. P. Lanyon, P. Zoller, R. Blatt, and C. F. Roos, Probing Entanglement Entropy via Randomized Measurements, Science 364, 260 (2019).

[11] J. Zeiher, R. van Bijnen, P. Schauß, S. Hild, J.-y. Choi, T. Pohl, I. Bloch, and C. Gross, Many-Body Interferometry of a Rydberg-Dressed Spin Lattice, Nat. Phys. 12, 1095 (2016).

[12] E. Guardado-Sanchez, P. T. Brown, D. Mitra, T. Devakul, D. A. Huse, P. Schauß, and W. S. Bakr, Probing the Quench Dynamics of Antiferromagnetic Correlations in a 2D Quantum Ising Spin System, Phys. Rev. X 8, 021069 (2018).

[13] D. Barredo, V. Lienhard, S. de Léséleuc, T. Lahaye, and A. Browaeys, Synthetic Three-Dimensional Atomic Structures Assembled Atom by Atom, Nature (London) 561, 79 (2018). 
[14] A. Keesling, A. Omran, H. Levine, H. Bernien, H. Pichler, S. Choi, R. Samajdar, S. Schwartz, P. Silvi, S. Sachdev, P. Zoller, M. Endres, M. Greiner, V. Vuletic, and M. D. Lukin, Probing Quantum Critical Dynamics on a Programmable Rydberg Simulator, Nature (London) 568, 207 (2019).

[15] J. Z. Blumoff, K. Chou, C. Shen, M. Reagor, C. Axline, R. T. Brierley, M. P. Silveri, C. Wang, B. Vlastakis, S. E. Nigg, L. Frunzio, M. H. Devoret, L. Jiang, S. M. Girvin, and R. J. Schoelkopf, Implementing and Characterizing Precise Multiqubit Measurements, Phys. Rev. X 6, 031041 (2016).

[16] R. Barends et al., Digitized Adiabatic Quantum Computing with a Superconducting Circuit, Nature (London) 534, 222 (2016).

[17] J. S. Otterbach et al., Unsupervised Machine Learning on a Hybrid Quantum Computer, arXiv:1712.05771.

[18] M. Gong et al., Genuine 12-qubit entanglement on a superconducting quantum processor, Phys. Rev. Lett. 122, 110501 (2019).

[19] P. Hosur, X.-L. Qi, D. A. Roberts, and B. Yoshida, Chaos in Quantum Channels, J. High Energy Phys. 02 (2016) 004.

[20] P. Calabrese and J. Cardy, Quantum Quenches in $1+1$ Dimensional Conformal Field Theories, J. Stat. Mech. (2016) 064003.

[21] A. Bohrdt, C. B. Mendl, M. Endres, and M. Knap, Scrambling and Thermalization in a Diffusive Quantum ManyBody System, New J. Phys. 19, 063001 (2017).

[22] C. W. von Keyserlingk, T. Rakovszky, F. Pollmann, and S. L. Sondhi, Operator Hydrodynamics, OTOCs, and Entanglement Growth in Systems without Conservation Laws, Phys. Rev. X 8, 021013 (2018).

[23] A. Nahum, S. Vijay, and J. Haah, Operator Spreading in Random Unitary Circuits, Phys. Rev. X 8, 021014 (2018).

[24] R. Fan, P. Zhang, H. Shen, and H. Zhai, Out-of-Time-Order Correlation for Many-Body Localization, Sci. Bull. 62, 707 (2017).

[25] Y. Huang, Y. Zhang, and X. Chen, Out-of-Time-Ordered Correlators in Many-Body Localized Systems, Ann. Phys. (Berlin) 529, 1600318 (2017).

[26] X. Chen, T. Zhou, D. A. Huse, and E. Fradkin, Out-of-TimeOrder Correlations in Many-Body Localized and Thermal Phases, Ann. Phys. (Berlin) 529, 1600332 (2017).

[27] S. Sachdev and J. Ye, Gapless Spin-Fluid Ground State in a Random Quantum Heisenberg Magnet, Phys. Rev. Lett. 70, 3339 (1993).

[28] A. Kitaev, A Simple Model of Quantum Holography. KITP Strings Seminar and Entanglement Program (2015).

[29] P. Hayden and J. Preskill, Black Holes as Mirrors: Quantum Information in Random Subsystems, J. High Energy Phys. 09 (2007) 120.

[30] S. H. Shenker and D. Stanford, Black Holes and the Butterfly Effect, J. High Energy Phys. 03 (2014) 067.

[31] J. Maldacena, S. H. Shenker, and D. Stanford, A Bound on Chaos, J. High Energy Phys. 08 (2016) 106.

[32] S. Banerjee and E. Altman, Solvable Model for a Dynamical Quantum Phase Transition from Fast to Slow Scrambling, Phys. Rev. B 95, 134302 (2017).

[33] D. A. Roberts and B. Yoshida, Chaos and Complexity by Design, J. High Energy Phys. 04 (2017) 121.
[34] S. Pappalardi, A. Russomanno, B. Žunkovič, F. Iemini, A. Silva, and R. Fazio, Scrambling and Entanglement Spreading in Long-Range Spin Chains, Phys. Rev. B 98, 134303 (2018).

[35] S. V. Syzranov, A. V. Gorshkov, and V. M. Galitski, Interaction-Induced Transition in the Quantum Chaotic Dynamics of a Disordered Metal, Ann. Phys. (Amsterdam) 405, 1 (2019).

[36] B. Swingle, G. Bentsen, M. Schleier-Smith, and P. Hayden, Measuring the Scrambling of Quantum Information, Phys. Rev. A 94, 040302(R) (2016).

[37] G. Zhu, M. Hafezi, and T. Grover, Measurement of ManyBody Chaos Using a Quantum Clock, Phys. Rev. A 94, 062329 (2016).

[38] H. Shen, P. Zhang, R. Fan, and H. Zhai, Out-of-Time-Order Correlation at a Quantum Phase Transition, Phys. Rev. B 96, 054503 (2017).

[39] B. Yoshida and N. Y. Yao, Disentangling Scrambling and Decoherence via Quantum Teleportation, Phys. Rev. X 9, 011006 (2019).

[40] N. Y. Yao, F. Grusdt, B. Swingle, M. D. Lukin, D. M. Stamper-Kurn, J. E. Moore, and E. A. Demler, Interferometric Approach to Probing Fast Scrambling, arXiv:1607 .01801

[41] J. Li, R. Fan, H. Wang, B. Ye, B. Zeng, H. Zhai, X. Peng, and J. Du, Measuring Out-of-Time-Order Correlators on a Nuclear Magnetic Resonance Quantum Simulator, Phys. Rev. X 7, 031011 (2017).

[42] M. Gärttner, J. G. Bohnet, A. Safavi-Naini, M. L. Wall, J. J. Bollinger, and A. M. Rey, Measuring Out-of-Time-Order Correlations and Multiple Quantum Spectra in a TrappedIon Quantum Magnet, Nat. Phys. 13, 781 (2017).

[43] B. Swingle and N. Yunger Halpern, Resilience of Scrambling Measurements, Phys. Rev. A 97, 062113 (2018).

[44] K. A. Landsman, C. Figgatt, T. Schuster, N. M. Linke, B. Yoshida, N. Y. Yao, and C. Monroe, Verified Quantum Information Scrambling, Nature (London) 567, 61 (2019).

[45] I. Bloch, J. Dalibard, and S. Nascimbène, Quantum Simulations with Ultracold Quantum Gases, Nat. Phys. 8, 267 (2012).

[46] F. Haake, Quantum Signatures of Chaos (Springer, New York, 2010), Vol. 54.

[47] C. Dankert, R. Cleve, J. Emerson, and E. Livine, Exact and Approximate Unitary 2-Designs and Their Application to Fidelity Estimation, Phys. Rev. A 80, 012304 (2009).

[48] Y. Nakata, C. Hirche, M. Koashi, and A. Winter, Efficient Quantum Pseudorandomness with Nearly TimeIndependent Hamiltonian Dynamics, Phys. Rev. X 7, 021006 (2017).

[49] A. Elben, B. Vermersch, M. Dalmonte, J. I. Cirac, and P. Zoller, Rényi Entropies from Random Quenches in Atomic Hubbard and Spin Models, Phys. Rev. Lett. 120, 050406 (2018).

[50] B. Vermersch, A. Elben, M. Dalmonte, J. I. Cirac, and P. Zoller, Unitary n-Designs via Random Quenches in Atomic Hubbard and Spin Models: Application to the Measurement of Rényi Entropies, Phys. Rev. A 97, 023604 (2018).

[51] A. Elben, B. Vermersch, C. F. Roos, and P. Zoller, Statistical Correlations between Locally Randomized Measurements: A Toolbox for Probing Entanglement in Many-Body Quantum States, Phys. Rev. A 99, 052323 (2019). 
[52] B. Collins and P. Śniady, Integration with Respect to the Haar Measure on Unitary, Orthogonal and Symplectic Group, Commun. Math. Phys. 264, 773 (2006).

[53] P. W. Brouwer and C. W. J. Beenakker, Diagrammatic Method of Integration over the Unitary Group, with Applications to Quantum Transport in Mesoscopic Systems, J. Math. Phys. (N.Y.) 37, 4904 (1996).

[54] E. Leviatan, F. Pollmann, J. H. Bardarson, D. A. Huse, and E. Altman, Quantum Thermalization Dynamics with Matrix-Product States, arXiv:1702.08894.

[55] X. Chen, T. Zhou, and C. Xu, Measuring the Distance between Quantum Many-Body Wave Functions, J. Stat. Mech. (2018) 073101.

[56] Here, we use $\operatorname{Tr}\left(W(t)^{2}\right)=2^{N}$.

[57] For all numerical simulations presented in this work, we consider $N_{u}$ independent samplings of the unitaries $u$ and $U$; i.e., we sample new pairs of unitaries $u$ and $U$ for each realization.

[58] D. M. Basko, I. L. Aleiner, and B. L. Altshuler, MetalInsulator Transition in a Weakly Interacting Many-Electron System with Localized Single-Particle States, Ann. Phys. (Amsterdam) 321, 1126 (2006).

[59] R. Nandkishore and D. A. Huse, Many-Body Localization and Thermalization in Quantum Statistical Mechanics, Annu. Rev. Condens. Matter Phys. 6, 15 (2015).
[60] M. Serbyn, Z. Papić, and D. A. Abanin, Local Conservation Laws and the Structure of the Many-Body Localized States, Phys. Rev. Lett. 111, 127201 (2013).

[61] D. A. Huse, R. Nandkishore, and V. Oganesyan, Phenomenology of Fully Many-Body-Localized Systems, Phys. Rev. B 90, 174202 (2014).

[62] A. Pal and D. A. Huse, Many-Body Localization Phase Transition, Phys. Rev. B 82, 174411 (2010).

[63] M. C. Tran, A. Y. Guo, Y. Su, J. R. Garrison, Z. Eldredge, M. Foss-Feig, A. M. Childs, and A. V. Gorshkov, Locality and Digital Quantum Simulation of Power-Law Interactions, arXiv:1808.05225.

[64] D. J. Luitz and Y. B. Lev, Emergent Locality in Systems with Power-Law Interactions, Phys. Rev. A 99, 010105 (2019).

[65] H. P. Breuer, E. M. Laine, and J. Piilo, Measure for the Degree of Non-Markovian Behavior of Quantum Processes in Open Systems, Phys. Rev. Lett. 103, 210401 (2009).

[66] J. R. Johansson, P. D. Nation, and F. Nori, QuTiP 2: A Python Framework for the Dynamics of Open Quantum Systems, Comput. Phys. Commun. 184, 1234 (2013).

[67] U. Schollwöck, The Density-Matrix Renormalization Group in the Age of Matrix Product States, Ann. Phys. (Amsterdam) 326, 96 (2011). 